\title{
A Strengthened Dominance Relation Considering Convergence and Diversity for Evolutionary Many-Objective Optimization
}

\author{
Ye Tian, Ran Cheng, Xingyi Zhang, Yansen Su, and Yaochu Jin, Fellow, IEEE
}

\begin{abstract}
Both convergence and diversity are crucial to evolutionary many-objective optimization, whereas most existing dominance relations show poor performance in balancing them, thus easily leading to a set of solutions concentrating on a small region of the Pareto fronts. In this paper, a novel dominance relation is proposed to better balance convergence and diversity for evolutionary manyobjective optimization. In the proposed dominance relation, an adaptive niching technique is developed based on the angles between the candidate solutions, where only the best converged candidate solution is identified to be nondominated in each niche. Experimental results demonstrate that the proposed dominance relation outperforms existing dominance relations in balancing convergence and diversity. A modified NSGA-II is suggested based on the proposed dominance relation, which shows competitiveness against the state-of-the-art algorithms in solving many-objective optimization problems. The effectiveness of the proposed dominance relation is also verified on several other existing multi- and many-objective evolutionary algorithms.
\end{abstract}

Index Terms-Many-objective optimization, evolutionary algorithm, convergence, diversity, Pareto dominance

\section{INTRODUCTION}

D URING the last two decades, the evolutionary algorithms have been verified to be suitable for solving multi-objective optimization problems (MOPs) due to their population based characteristics [1]. Thanks to the success of NSGA-II [2], most existing multiobjective evolutionary algorithms (MOEAs) were developed based on the Pareto dominance relation, which was suggested by Goldberg for distinguishing the quality of

Manuscript received -. This work was supported in part by the National Natural Science Foundation of China under Grant 61272152, Grant 61502004, Grant 61502001 and Grant 61672033. The work of Y. Jin was supported in part by the U.K. EPSRC under Grant EP/M017869/1. (Corresponding author: Xingyi Zhang)

Y. Tian is with the Institute of Physical Science and Information Technology, Anhui University, Hefei 230601, China (email: field910921@gmail.com)

R. Cheng is with the Shenzhen Key Laboratory of Computational Intelligence, Department of Computer Science and Engineering, Southern University of Science and Technology, Shenzhen 518055, China. (email: ranchengcn@gmail.com)

$\mathrm{X}$. Zhang and Y. Su are with the Institute of Bio-inspired Intelligence and Mining Knowledge, School of Computer Science and Technology, Anhui University, Hefei 230601, China (email: xyzhanghust@gmail.com; suyansen1985@163.com).

Y. Jin is with the Department of Computer Science, University of Surrey, Guildford, Surrey, GU2 7XH, U.K. (email: yaochu.jin@surrey.ac.uk). candidate solutions [3]. However, the performance of most Pareto dominance based MOEAs suffers a serious curse of dimensionality in solving MOPs with more than three objectives, i.e., the many-objective optimization problems (MaOPs) [4]. In many-objective optimization, the challenge can be mainly attributed to a phenomenon known as the dominance resistance [5], where most candidate solutions become incomparable in the sense of Pareto dominance. To address this issue, a variety of methods have been proposed to enhance Pareto dominance for solving MaOPs, which can be roughly divided into the following two categories.

The first category focuses on developing new dominance relations, where the basic idea is to increase the probability that two candidate solutions are comparable on MaOPs. There exist many techniques for developing new dominance relations in the literature, such as expanding the dominance area [6], [7], gridding the objective space [8], [9], adopting the fuzzy logic [10], [11], and defining the dominance relation by weight vectors [12], [13].

The second category is characterized by combining Pareto dominance with additional selection criteria. These methods first eliminate a few worse candidate solutions based on Pareto dominance, then distinguish the incomparable candidate solutions by the secondary selection criterion. Existing methods belonging to this category mainly adopt three basic ideas: The first idea is to develop new criterion to bias those with good convergence and diversity in the incomparable candidate solutions, such as KnEA [14] and VaEA [15]; the second idea employs performance indicators to distinguish the quality of incomparable candidate solutions by selecting those with large contributions, such as HypE [16] and AR-MOEA [17]; and the third idea is to combine Pareto dominance with decomposition based algorithms, such as MOEA/DD [18] and BCE-MOEA/D [19].

While the modified dominance relations can generate relatively higher selection pressure for MOEAs than the conventional Pareto dominance relation, most of them fail to strike a good balance between convergence and diversity, which easily leads to a set of solutions concentrating on a small region of the Pareto fronts. Hence, in this paper we propose a novel dominance relation 
for MOEAs to solve MaOPs, which can better balance convergence and diversity than existing dominance relations. The contributions of this paper are summarized as follows:

1) A novel dominance relation, termed strengthened dominance relation (SDR), is proposed for evolutionary many-objective optimization. The proposed SDR balances convergence and diversity by developing a niching technique based on the angles between the candidate solutions, and maintains only one candidate solution with the best convergence in each niche. Since the niche size is adaptively determined by the distribution of the candidate solutions measured by the angles between them, the proposed SDR is parameterless to use.

2) An MOEA, named NSGA-II/SDR, is suggested by embedding SDR in NSGA-II. The NSGA-II/SDR holds the same framework with NSGA-II, where the conventional Pareto dominance relation is replaced by the proposed SDR. The performance of NSGA-II/SDR is verified by comparing it with several state-of-the-art MOEAs tailored for solving $\mathrm{MaOPs}$

3) The performance of the proposed SDR is also assessed by embedding it in two existing MOEAs developed for solving MOPs, namely, SPEA2 [20] and PESA-II [21], and three existing MOEAs tailored for handling MaOPs, namely, GrEA [9], KnEA [14], and VaEA [15]. Experimental results demonstrate that the proposed SDR maintains a good balance between convergence and diversity, which can considerably improve the performance of the MOEAs.

In the remainder of the paper, we first give a review of existing dominance relations in Section II. Then the proposed SDR is elaborated in Section III and the experimental results are given in Section IV. Finally, conclusions are drawn in Section V.

\section{RELATED WORK}

\section{A. Existing Dominance Relations}

The Pareto dominance relation is widely adopted in MOEAs for distinguishing the quality of candidate solutions [22], [23]. For a minimization MOP, a candidate solution $\mathbf{x}$ is said to Pareto dominate another one $\mathbf{y}$, denoted as $\mathbf{f}(\mathbf{x}) \prec \mathbf{f}(\mathbf{y})$, if and only if

$$
\left\{\begin{array}{l}
\forall i \in 1, \ldots, M: f_{i}(\mathbf{x}) \leq f_{i}(\mathbf{y}) \\
\exists j \in 1, \ldots, M: f_{j}(\mathbf{x})<f_{j}(\mathbf{y})
\end{array},\right.
$$

where $\mathbf{f}(\mathbf{x})=\left(f_{1}(\mathbf{x}), f_{2}(\mathbf{x}), \ldots, f_{M}(\mathbf{x})\right)$ denotes the objective values of $\mathrm{x}$ and $M$ is the number of objectives. If neither $\mathbf{x}$ dominates $\mathbf{y}$ nor $\mathbf{y}$ dominates $\mathbf{x}$, the two candidate solutions are known to be incomparable or non-dominated to each other.

According to (1), for two random candidate solutions with $M$ objectives, the probability that one candidate solution dominates the other one is $1 / 2^{M-1}$, where the probability will exponentially decrease as the number of objectives $M$ increases, and such a phenomenon is known as the dominance resistance [5]. To address this issue, a variety of approaches have been proposed to increase the probability that two candidate solutions are distinguishable, which can be roughly grouped into four categories. Fig. 1 illustrates some representative dominance relations by plotting the dominance areas in bi- and three-objective spaces. In the following, we briefly review the representative dominance relations in the four categories.

The first category of dominance relations improves the selection pressure by expanding the dominance area. The controlling dominance area of solutions (CDAS) method [6] is a representative one falling into this category, which expands the dominance area of a candidate solution $\mathrm{x}$ by modifying the objective values:

$$
f_{i}^{\prime}(\mathbf{x})=\frac{\|\mathbf{f}(\mathbf{x})\| \sin \left(\omega_{i}+S \cdot \pi\right)}{\sin (S \cdot \pi)}, i=1, \ldots, M,
$$

where $f_{i}^{\prime}(\mathbf{x})$ denotes the $i$-th objective value of $\mathbf{x}$ after modification, $\|\cdot\|$ denotes the $L_{2}$-norm, $\omega_{i}$ denotes the declination angle between $\mathrm{x}$ and the $i$-th axis, and $S \in$ $[0.25,0.5]$ is a parameter for controlling the expanding degree.

In order to eliminate the parameter $S$, an adaptive version of CDAS, called self-CDAS (S-CDAS), was proposed [24]. The S-CDAS adaptively determines the expanding degree of a candidate solution $\mathrm{x}$ according to the extreme solutions in the population:

$$
f_{i}^{\prime}(\mathbf{x})=\frac{\|\mathbf{f}(\mathbf{x})\| \sin \left(\omega_{i}+\phi_{i}\right)}{\sin \left(\phi_{i}\right)}, i=1, \ldots, M
$$

where

$$
\phi_{i}=\arcsin \frac{\|\mathbf{f}(\mathbf{x})\| \cdot \sin \left(\omega_{i}\right)}{\left\|\mathbf{f}(\mathbf{x})-p_{i}\right\|}
$$

and $p_{i}$ is the extreme solution with respect to the $i$-th axis in the population. As shown in Fig. 1, the boundary of the dominance area of $\mathrm{x}$ in the sense of S-CDAS always intersects (but does not dominate) the extreme solutions.

Another idea for expanding the dominance area is to modify the definition of dominance relation, such as $\alpha$-dominance [25] and generalized Pareto optimality (GPO) [7]. In GPO, a candidate solution $\mathrm{x}$ dominates another candidate solution $\mathbf{y}$ if and only if the following condition holds:

$$
\left\{\begin{array}{l}
\forall i \in 1, \ldots, M: f_{i}(\mathbf{x})-f_{i}(\mathbf{y}) \leq \sum_{k \neq i} \delta_{i}\left(f_{k}(\mathbf{y})-f_{k}(\mathbf{x})\right) \\
\exists j \in 1, \ldots, M: f_{j}(\mathbf{x})-f_{j}(\mathbf{y})<\sum_{k \neq j} \delta_{j}\left(f_{k}(\mathbf{y})-f_{k}(\mathbf{x})\right)
\end{array},\right.
$$

where $\delta_{i}=\sqrt{M-1} \cdot \tan \phi_{i} /(M-1)$ and $\phi_{i}$ is a parameter of the expanding angle on the $i$-th objective. As reported in [7], while CDAS and GPO are equivalent in bi-objective space, GPO can guarantee the identity of dominance envelopes (i.e., the boundaries of dominance area) in three- and higher-objective spaces.

The second category of dominance relations is based on the gridding of the objective space, such as $\epsilon$-dominance [8], pat-dominance [26], cone $\epsilon$ dominance [27], and grid dominance [9]. In the 


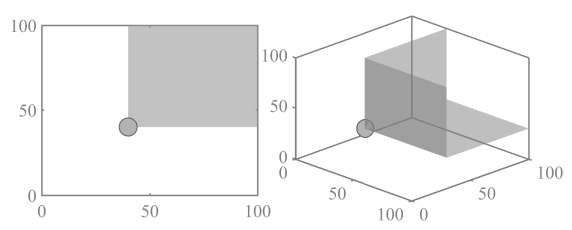

(a) Pareto dominance

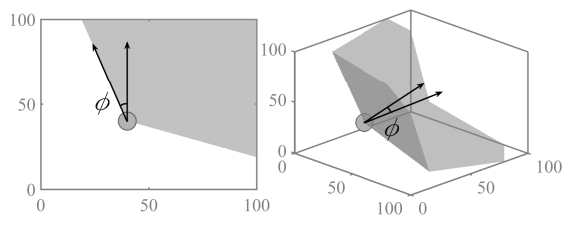

(d) Generalized Pareto optimality (GPO)

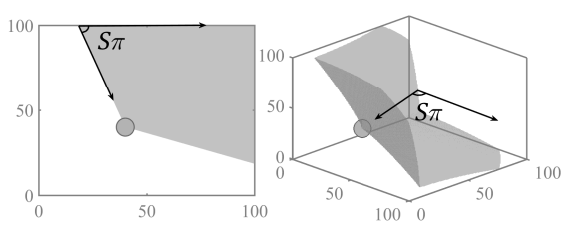

(b) Controlling dominance area of solutions (CDAS)

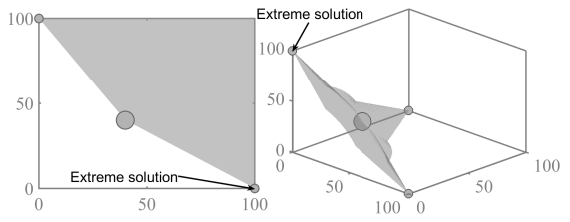

(c) Self-CDAS (S-CDAS)

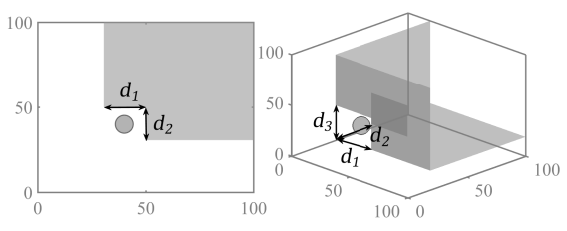

(e) Grid dominance

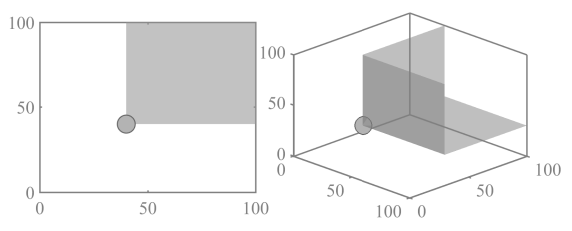

(f) $(1-k)$-dominance

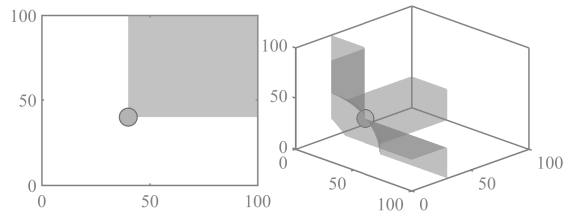

(g) L-dominance

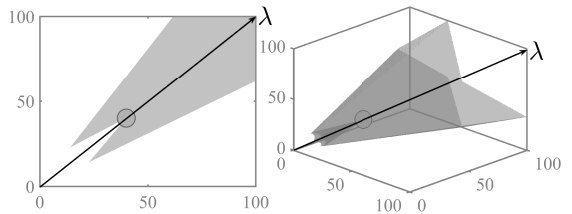

(h) $\theta$-dominance

Fig. 1. The dominance areas obtained by eight different dominance relations in bi- and three-objective spaces.

grid dominance relation, the grid coordinate $\mathbf{g}(\mathbf{x})=$ $\left(g_{1}(\mathbf{x}), g_{2}(\mathbf{x}), \ldots, g_{M}(\mathbf{x})\right)$ of candidate solution $\mathbf{x}$ is calculated by

$$
g_{i}(\mathbf{x})=\left\lfloor\left(f_{i}(\mathbf{x})-l b_{i}\right) / d_{i}\right\rfloor,
$$

and

$$
\begin{aligned}
d_{i} & =\left(u b_{i}-l b_{i}\right) / \operatorname{div} \\
u b_{i} & =\max f_{i}+\left(\max f_{i}-\min f_{i}\right) / \operatorname{div} / 2, \\
l b_{i} & =\min f_{i}-\left(\max f_{i}-\min f_{i}\right) / \operatorname{div} / 2
\end{aligned}
$$

where $\min f_{i}$ and $\max f_{i}$ denote the minimum and maximum values of the $i$-th objective in the population, div is a parameter of the number of divisions on each objective, and the grid coordinates are used to determine the dominance relations instead of the objective values. The grid dominance relation is a relaxation of Pareto dominance, whereas it is often criticized due to its high sensitivity to the parameter div [9].

The third category adopts fuzzy logic to define new dominance relations, such as (1-k)-dominance [28], Ldominance [29], and fuzzy dominance [11]. In these dominance relations, the number of objectives where one candidate solution is smaller or greater than another is usually considered as the criterion to determine the dominance relation. For the (1-k)-dominance relation, a candidate solution $\mathrm{x}$ is said to $(1-k)$-dominate another candidate solution $\mathbf{y}$ if and only if

$$
\left\{\begin{array}{l}
n_{e}<M \\
n_{l} \geq \frac{M-n_{e}}{k+1}
\end{array},\right.
$$

where $n_{e}$ and $n_{l}$ count the number of objectives where $\mathbf{x}$ is equal to and smaller than $\mathbf{y}$, respectively, and $k \in[0,1]$ is a predefined parameter. For the L-dominance relation, $\mathbf{x}$ is said to dominate $\mathbf{y}$ if and only if

$$
\left\{\begin{array}{c}
n_{l}>n_{g} \\
\|\mathbf{f}(\mathbf{x})\|<\|\mathbf{f}(\mathbf{y})\|
\end{array}\right.
$$

where $n_{l}$ and $n_{g}$ count the number of objectives where $\mathbf{x}$ is smaller and greater than $\mathbf{y}$, respectively. As shown in Fig. 1, (1-k)-dominance and L-dominance hold the same dominance areas with Pareto dominance in biobjective space, whereas the difference between $(1-k)$ dominance and Pareto dominance only exists when the number of objectives $M \geq 4$, and the difference between L-dominance and Pareto dominance only exists when $M \geq 3$,

Inspired by decomposition based MOEAs, the fourth category of dominance relations is defined by a set of weight vectors, e.g., the $\theta$-dominance relation [12] and the RP-dominance relation [13]. In $\theta$-dominance, each candidate solution is associated with its nearest weight vector, and a candidate solution $\mathbf{x}$ is said to $\theta$-dominate another one $\mathbf{y}$ if and only if they are associated with the same weight vector $\lambda$, and

$$
d_{1}(\mathbf{x}, \lambda)+\theta \cdot d_{2}(\mathbf{x}, \lambda)<d_{1}(\mathbf{y}, \lambda)+\theta \cdot d_{2}(\mathbf{y}, \lambda),
$$

where $\theta$ is a penalty parameter and

$$
\begin{aligned}
& d_{1}(\mathbf{x}, \lambda)=\left\|\mathbf{f}(\mathbf{x}) \lambda^{T}\right\| /\|\lambda\| \\
& d_{2}(\mathbf{x}, \lambda)=\left\|\mathbf{f}(\mathbf{x})-d_{1}(\mathbf{x}, \lambda)(\lambda /\|\lambda\|)\right\| .
\end{aligned}
$$

The $\theta$-dominance relation guides the candidate solutions to converge along the weight vectors, and thus maintains a uniform distribution in the population. However, since the population size should be identical to the number of weight vectors, the performance will substantially deteriorate if the distribution of the weight vectors is inconsistent with the shape of the Pareto front [30]. 


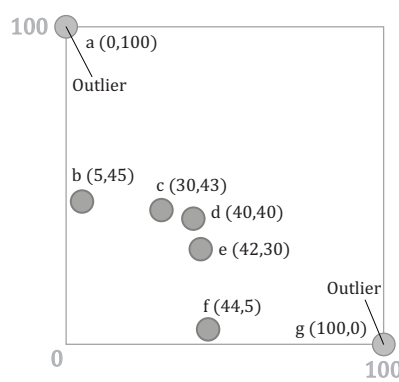

(a) A population with seven candidate solutions

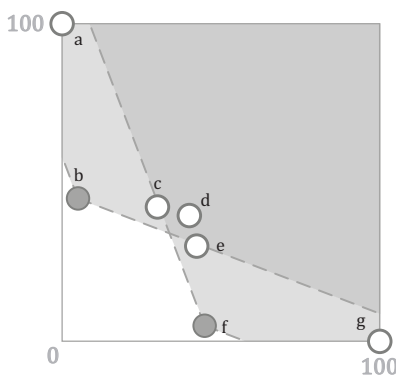

(c) CDAS and GPO

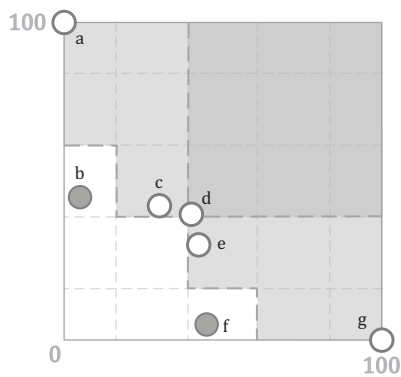

(e) Grid dominance

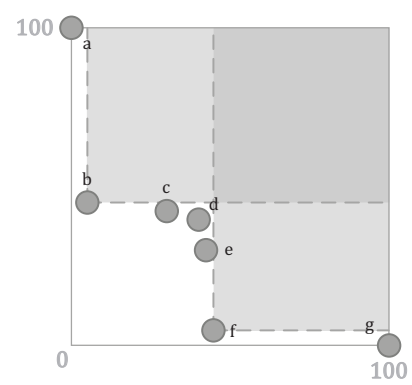

(b) Pareto dominance, $(1-k)$ dominance, and L-dominance

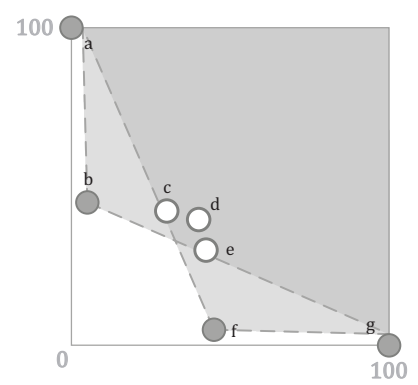

(d) S-CDAS

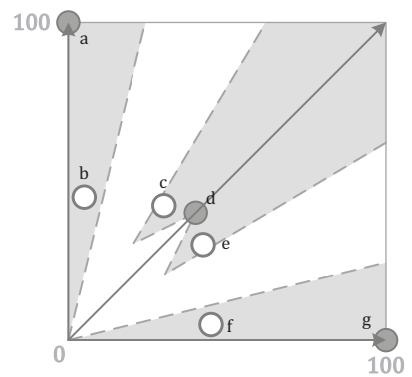

(f) $\theta$-dominance
Fig. 2. The results of eight dominance relations performed on a population with seven candidate solutions, where the filled circles denote non-dominated solutions identified by the dominance relations, the empty circles denote dominated solutions, and the gray regions denote dominance areas.

\section{B. Analysis of Existing Dominance Relations}

As introduced above, most existing dominance relations aim to enhance the selection pressure of MOEAs, but they can fail to achieve a good balance between convergence and diversity. Nevertheless, a balanced convergence and diversity is crucial to the performance of MOEAs in solving MaOPs. To further investigate the capabilities of existing dominance relations on this aspect, we consider the population containing seven candidate solutions as shown in Fig. 2(a). In this population, candidate solutions $\mathbf{b}, \mathbf{c}, \mathbf{d}, \mathbf{e}$, and $\mathbf{f}$ have much smaller objective values than candidate solutions a and g. In terms of convergence and diversity, it is clear that candidate solutions $\mathbf{a}$ and $\mathbf{g}$ should be identified as dominated solutions and discarded, whereas the other candidate solutions should be identified as non-dominated solutions. Since the Pareto front is not known a priori, the convergence is determined by the summation of objective values of a population rather than the distance of the population to the Pareto front. Therefore, the convergence of the non-dominated solution set will substantially deteriorate if candidate solutions $\mathbf{a}$ and $\mathbf{g}$ are selected. On the other hand, the definition of diversity is consistent with those in most literature (i.e., evenness and spread of a population [31], [32]), so the diversity of the non-dominated solution set can only be improved a little if $\mathbf{a}$ and $\mathbf{g}$ are selected.

All the eight dominance relations shown in Fig. 1 are employed to identify the non-dominated solutions in the population, and the parameters of them follow the settings as suggested in their original literature. To be specific, the parameters $S$ in CDAS is set to 0.39 , the parameter $\phi_{i}$ in GPO is set to 0.34 , the parameter div in grid dominance is set to 5 , the parameter $k$ in $(1-k)$ dominance is set to 0.25 , the number of reference vectors used by $\theta$-dominance is set to 3 , and the parameter $\theta$ in $\theta$-dominance is set to $10^{6}$ for weight vectors on the axes and 5 for the rest weight vectors.

The results are shown in Fig. 2(b)-(f), where the filled circles denote non-dominated solutions identified by the dominance relations, the empty circles denote dominated solutions, and the gray regions denote dominance areas. As shown in Fig. 2(b), Pareto dominance, (1$k$ )-dominance, and L-dominance identify all the seven candidate solutions as non-dominated solutions, since their dominance areas are identical in bi-objective space. By expanding the dominance area, CDAS and GPO identify $\mathbf{a}$ and $\mathbf{g}$ as dominated solutions as shown in Fig. 2(c), while they also discard c, d, and e. Regarding $\mathrm{S}$-CDAS, since the dominance area is determined by the extreme solutions a and g, S-CDAS cannot discard the two candidate solutions; by contrast, S-CDAS identifies $\mathbf{c}, \mathbf{d}$, and $\mathbf{e}$ as dominated solutions. The result of grid dominance relation is similar to those of CDAS and GPO, where candidate solutions $\mathbf{a}, \mathbf{c}, \mathbf{d}, \mathbf{e}$, and $\mathbf{g}$ are identified to be dominated by candidate solutions $\mathbf{b}$ and f according to their grid coordinates. For $\theta$-dominance, the three candidate solutions $\mathbf{a}, \mathbf{d}$, and $\mathbf{g}$ that are close to the weight vectors are identified as non-dominated solutions.

To summarize, for the population shown in Fig. 2(a), Pareto dominance, (1-k)-dominance, L-dominance, and $\theta$-dominance lead to poor convergence and good diversity; CDAS, GPO, and grid dominance lead to good convergence and poor diversity; S-CDAS leads to both poor convergence and diversity; whereas none of them can well balance convergence and diversity in the obtained non-dominated solution set. Therefore, to address this issue, we propose a new dominance relation in this work. The proposed domination relation can not only enhance the selection pressure of MOEAs, but also maintain a good balance between convergence and diversity. 


\section{StRengthened Dominance Relation}

\section{A. The Proposed Dominance Relation}

The proposed strengthened dominance relation (SDR) balances the convergence and diversity of the nondominated solution set by adopting a tailored niching technique. Specifically, a candidate solution $\mathrm{x}$ is said to dominate another candidate solution $y$ in SDR (denoted as $\mathbf{x} \prec_{S D R} \mathbf{y}$ ) if and only if

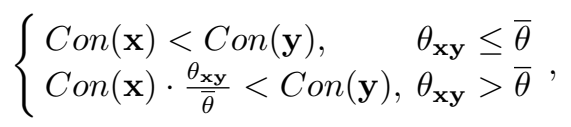

where

$$
\operatorname{Con}(\mathbf{x})=\sum_{i=1}^{M} f_{i}(\mathbf{x})
$$

is a metric for measuring the convergence degree of $\mathrm{x}$, which has been widely used in many MOEAs [15], [33][35], $\theta_{\mathbf{x y}}$ denotes the acute angle between the objective values of the two candidate solutions, namely,

$$
\theta_{\mathbf{x y}}=\arccos (\mathbf{f}(\mathbf{x}), \mathbf{f}(\mathbf{y}))
$$

and $\bar{\theta}$ is the size of the niche that each candidate solution belongs to. In order to better handle MaOPs whose objectives are badly-scaled or where the ideal point is not the origin, the objective values of all candidate solutions should be normalized according to the ideal point and nadir point of the Pareto front before calculating $\operatorname{Con}(\mathbf{x})$ and $\theta_{\mathbf{x y}}$. However, since the Pareto front is not known a priori, the minimum value of each objective and the maximum value of each objective of the population are adopted as the ideal point and nadir point, respectively.

As can be seen from (12), each candidate solution $\mathbf{x}$ in the population has a niche with size $\bar{\theta}$, and the dominance relationship related to the candidate solution $\mathbf{x}$ is determined by mainly considering the candidate solutions in its niche. According to the first formula in (12), if the angle between any $\mathrm{x}$ and a candidate solution $\mathbf{y}$ is smaller than $\bar{\theta}, \mathbf{x}$ is said to dominate $\mathbf{y}$ once the convergence degree of $\mathrm{x}$ is smaller than that of $\mathrm{y}$. It means that, inside each niche, since there do not exist two non-dominated solutions having a smaller angle than $\bar{\theta}$, the diversity of the non-dominated solution set is naturally preserved. By contrast, according to the second formula in (12), if two candidate solutions $\mathbf{x}$ and $\mathbf{y}$ are not inside the same niche (i.e., $\theta_{\mathbf{x y}}>\bar{\theta}$ ), $\mathbf{x}$ is still able to dominate $\mathbf{y}$ if the convergence degree of $\mathbf{y}$ is much worse than that of $\mathrm{x}$, where the probability that $\mathrm{x}$ dominates $\mathbf{y}$ is negatively related with the angle $\theta_{\mathbf{x y}}$. In this way, the convergence of the non-dominated solution set can be guaranteed.

Fig. 3 illustrates the dominance area obtained by SDR in bi-objective space. On one hand, since $\mathbf{y}_{\mathbf{1}}$ is located inside the niche of $\mathrm{x}$ and has worse convergence degree than $\mathbf{x}, \mathbf{x}$ dominates $\mathbf{y}_{\mathbf{1}}$; on the other hand, since $\mathbf{y}_{\mathbf{2}}$ is located outside the niche of $\mathbf{x}$ and has much worse convergence degree than $\mathrm{x}, \mathrm{x}$ still dominates $\mathrm{y}_{2}$. As a result, the dominance area of $\mathrm{x}$ consists of two parts:

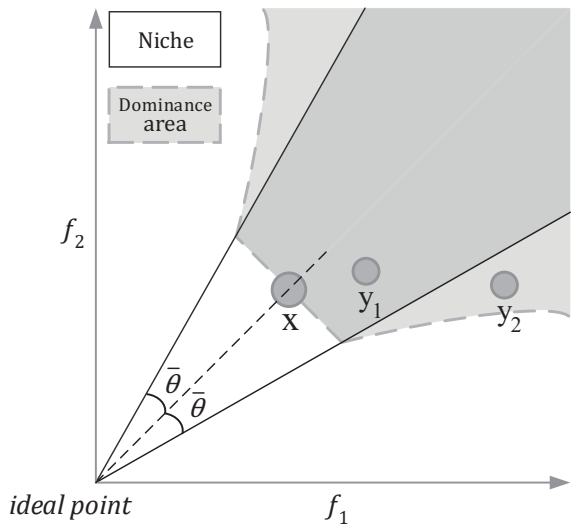

Fig. 3. The dominance area of solution $x$ obtained by SDR in biobjective space. $\mathbf{x}$ dominates $\mathbf{y}_{1}$ since $\mathbf{y}_{1}$ is located inside the niche of $\mathbf{x}$ (i.e., $\theta_{\mathbf{x y}_{1}}<\bar{\theta}$ ) and has worse convergence degree than $\mathbf{x} ; \mathbf{x}$ dominates $\mathbf{y}_{\mathbf{2}}$ since $\mathbf{y}_{\mathbf{2}}$ is located outside the niche of $\mathbf{x}$ (i.e., $\theta_{\mathbf{x y}_{2}}>\bar{\theta}$ ) and has rather worse convergence degree than $\mathbf{x}$.

The first part is given by the first formula in (12), where $\mathbf{x}$ dominates all candidate solutions inside its niche with worse convergence degrees than it; the second part is given by the second formula in (12), where $\mathrm{x}$ dominates those outside its niche with rather worse convergence degrees than it. According to (12), it is worth noting that some candidate solutions that are worse in all objectives compared to another solution (i.e., the candidate solutions that are Pareto-dominated) may be regarded as non-dominated by SDR, which is not desired. However, SDR does not adopt Pareto dominance to regard these solutions as dominated, since only a few candidate solutions in the population are Pareto-dominated on MaOPs, and they have little influence on the performance of SDR. More importantly, the detection of these solutions can highly increase the computational complexity of SDR.

The parameter $\bar{\theta}$, which determines the niche size, is crucial to the proposed SDR. While similar parameters in existing dominance relations such as CDAS and GPO are predefined by users, the parameter $\bar{\theta}$ in the proposed SDR can be adaptively estimated according to the distribution of candidate solution set. For most existing MOEAs, since the environmental selection always selects half of the combined population obtained at each generation, the target of adapting $\bar{\theta}$ is to guarantee that the ratio of the non-dominated solutions in a given candidate solution set is around 0.5. Consider the two populations shown in Fig. 4 of different distributions, $\bar{\theta}$ should be set to 0.3 if three candidate solutions in each population are expected to be dominated since only one candidate solution is maintained in each niche. Specifically, for the population shown on the left, the minimum angles between each candidate solution and the rest are $0.1,0.1,0.2,0.2,0.3$ and 0.3 , hence either one of $\mathbf{a}$ and $\mathbf{b}, \mathbf{c}$ and $\mathbf{d}$, or $\mathbf{e}$ and $\mathbf{f}$ can be dominated; for the population shown on the right, the minimum angles between each candidate solution and the rest are $0.1,0.1$, $0.2,0.3,0.4$ and 0.5 , hence at most three of $\mathbf{a}, \mathbf{b}, \mathbf{c}$, and 


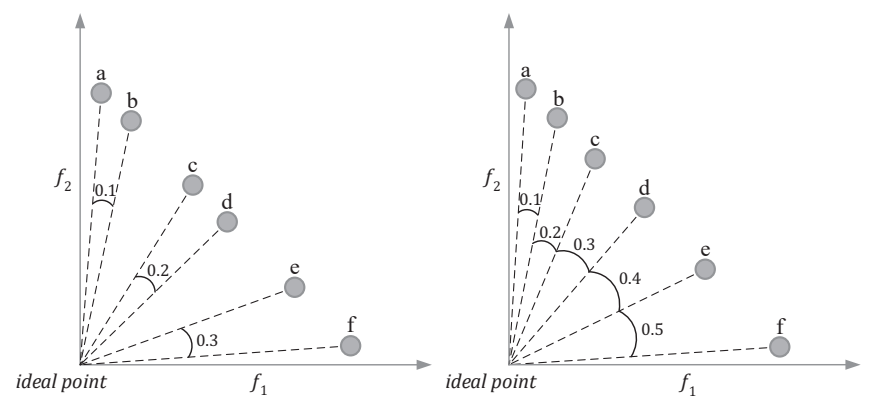

Fig. 4. Two populations of different distributions, where the minimum angles between each candidate solution and the rest in the population shown on the left are $0.1,0.1,0.2,0.2,0.3$ and 0.3 , and the minimum angles between each candidate solution and the rest in the population shown on the right are $0.1,0.1,0.2,0.3,0.4$ and 0.5 . Obviously, $\theta$ should be set to 0.3 if the number of non-dominated solutions is expected to be half the population size since only one candidate solution is maintained in each niche.

d can be dominated. In fact, $\bar{\theta}$ should be set to the third minimum value of the unique values of the minimum angles between each pair of candidate solutions, which is exactly 0.3 for both of the two populations. As a result, in order to guarantee that the ratio of non-dominated solutions in a solution set $P$ is always around $0.5, \bar{\theta}$ is set to the $\left\lfloor\frac{|P|}{2}\right\rfloor$-th minimum element of

$$
\left\{\min _{\mathbf{q} \in P \backslash\{\mathbf{p}\}} \theta_{\mathbf{p q}} \mid \mathbf{p} \in P\right\},
$$

where $\theta_{\mathbf{p q}}$ denotes the acute angle between any pair of candidate solutions $\mathbf{p}$ and $\mathbf{q}$. Some empirical results demonstrating the effectiveness of adaptive estimation of $\bar{\theta}$ can be found in Section IV-E.

We have the following properties according to the definition of SDR, which illustrate that SDR is irreflexive, antisymmetric, and non-transitive, respectively.

Property 1: For any candidate solution $\mathbf{x}, \mathbf{x} \nprec_{S D R} \mathbf{x}$.

Proof: According to (12), if $\mathbf{x} \prec_{S D R} \mathbf{y}$, then $\operatorname{Con}(\mathbf{x})<$ $\operatorname{Con}(\mathbf{y})$. Since $\operatorname{Con}(\mathbf{x}) \nless C$ on $(\mathbf{x}), \mathbf{x} \nprec_{S D R} \mathbf{x}$.

Property 2: If $\mathbf{x} \prec_{S D R} \mathbf{y}$, then $\mathbf{y} \nprec_{S D R} \mathbf{x}$.

Proof: By $\mathbf{x} \prec S D R \mathbf{y}$, we have that $\operatorname{Con}(\mathbf{x})<\operatorname{Con}(\mathbf{y})$, i.e., $\operatorname{Con}(\mathbf{y}) \nless \operatorname{Con}(\mathbf{x})$, so $\mathbf{y} \nprec_{S D R} \mathbf{x}$.

Property 3: If $\mathbf{x} \prec_{S D R} \mathbf{y}$ and $\mathbf{y} \prec_{S D R} \mathbf{z}$, we cannot deduce that $\mathbf{x} \prec_{S D R} \mathbf{z}$.

Proof: Assume that $\mathbf{f}(\mathbf{x})=(0,3), \mathbf{f}(\mathbf{y})=(2,2)$, $\mathbf{f}(\mathbf{z})=(5,0)$, and $\bar{\theta}=\pi / 4$. According to (12), we have that $\mathbf{x} \prec_{S D R} \mathbf{y}, \mathbf{y} \prec_{S D R} \mathbf{z}$, but $\mathbf{x} \nprec_{S D R} \mathbf{z}$.

Note that since the proposed SDR is non-transitive, some popular non-dominated sorting approaches like deductive sort [36] and efficient non-dominated sort [22] cannot be adopted. This is because these non-dominated sorting approaches take advantage of the transitivity of dominance relation, and some dominated solutions may be regarded as non-dominated if the dominance relation is non-transitive. As a consequence, SDR can only be embedded in the non-dominated sorting approaches that do not take advantage of the transitivity of dominance relation, such as fast non-dominated sort [2].

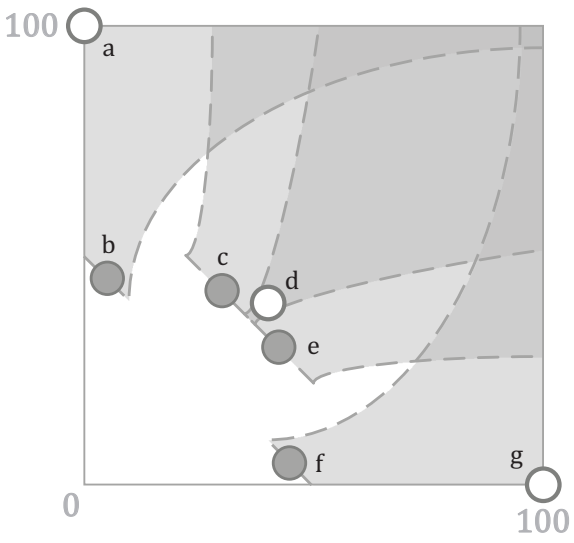

Fig. 5. The result of SDR performed on a population with seven candidate solutions, where the filled circles denote non-dominated solutions identified by SDR, the empty circles denote dominated solutions, and the gray regions denote dominance areas.

\section{B. Analysis of SDR}

In the following, we verify the effectiveness of SDR in balancing convergence and diversity by comparing it with eight existing dominance relations shown in Fig. 1. We first consider the proposed SDR on the population shown in Fig. 2(a). The non-dominated solutions identified by SDR are plotted in Fig. 5, where SDR identifies $\mathbf{b}, \mathbf{c}$, $\mathbf{e}$, and $\mathbf{f}$ as non-dominated solutions. $\mathbf{a}$ and $\mathbf{g}$ are identified as dominated solutions by SDR since they have much worse convergence degrees than the others, and $\mathbf{d}$ is identified as dominated solution since it locates in the same niche with $\mathbf{c}$ or e. It is clear that the nondominated solution set identified by SDR has better convergence and diversity than those identified by the dominance relations shown in Fig. 2.

Despite that existing dominance relations can enhance selection pressure for MOEAs in solving MaOPs, as shown in the literature [7], [8], [30], most of the dominance relations can only find a set of solutions concentrating on a small region of the Pareto front. The Pareto front of an MOP consists of all the nondominated solutions in the objective space in the sense of Pareto dominance, and MOEAs aim to find a representative set of solutions covering the Pareto front as comprehensively as possible. However, since most existing dominance relations are modified to be more strict than the original Pareto dominance relation, some non-dominated solutions on the Pareto front can also be identified as dominated, thus sacrificing the distribution of the candidate solutions. By contrast, as evidenced by Fig. 6, the proposed SDR can address this issue as it maintains a good balance between convergence and diversity, where the non-dominated regions are generated using the non-dominated solutions identified by each dominance relation among 5000 solutions randomly sampled on each Pareto front. Note that the Pareto dominance relation and (1-k)-dominance relation are not involved in the figure, since these two dominance relations will identify all the candidate solutions on the 


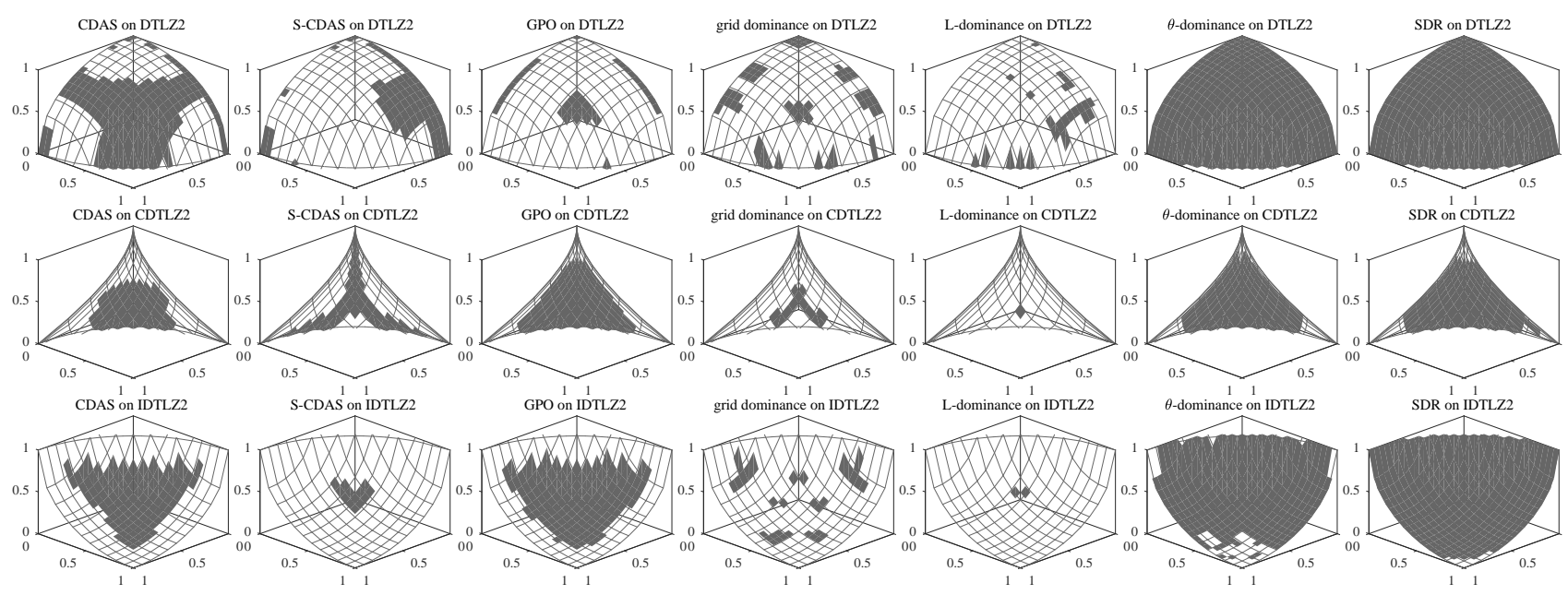

Fig. 6. The non-dominate regions (shown in gray) identified by seven dominance relations on the Pareto fronts of DTLZ2, CDTLZ2, and IDTLZ2 with 3 objectives, which are generated using the non-dominated solutions identified by each dominance relation among 5000 solutions randomly sampled on each Pareto front.

Pareto fronts as non-dominated solutions if the number of objectives is smaller than four.

The parameter settings of these dominance relations are the same to the recommended ones as described in Section II-B. The number of reference vectors used in $\theta$ dominance is set to 300. As can be observed from Fig. 6, the non-dominated region identified by SDR roughly covers the whole Pareto front of each test instance, whereas the non-dominated regions identified by the other existing dominance relations easily shrink to a small region of each Pareto front. To be specific, for dominance area expansion based relations, CDAS, S-CDAS, and GPO, the non-dominated regions identified by them are merely located in the center of each Pareto front, since the candidate solutions in the center can dominate the boundary candidate solutions in terms of the expanded dominance area. The non-dominated region identified by grid dominance uniformly distributes in several small regions of each Pareto front, failing to comprehensively cover the whole Pareto front. The is due to the fact that the grids in the grid dominance uniformly distribute in an $M$-dimensional hypercube, whereas the Pareto front is an $(M-1)$-dimensional manifold [37], and thus the grids intersecting with the Pareto front are discrete. For L-dominance, it identifies non-dominated solutions mainly based on their Euclidean distances to the origin in three-objective space, hence only the region closest to the origin can be identified as non-dominated region. For $\theta$-dominance, since the distribution of the weight vectors are uniformly sampled on a simplex unit hyperplane, the non-dominated region is able to cover the simplex-like Pareto fronts of DTLZ2 and CDTLZ2; however, it can not deal with irregular Pareto fronts where the shapes are not consistent with the uniformly sampled weight vectors, such as the inverted Pareto front of IDTLZ2.

By contrast, since the proposed SDR balances the convergence and diversity of the non-dominated solutions by identifying one candidate solution to be nondominated in each niche, it is always able to roughly cover the whole Pareto fronts of the various test instances. It should be noted that although the nondominated regions identified by SDR are similar to those identified by the Pareto dominance, SDR identifies some crowded solutions on the Pareto fronts as dominated solutions. The elimination of these crowded solutions has little influence on the convergence to the entire Pareto front, but can greatly improve the selection pressure on MaOPs.

SDR balances the convergence and diversity of the non-dominated solution set by keeping one solution with the best convergence degree in each niche. In fact, scalarizing multicriteria approaches (e.g., hyperbola efficiency [38]) and decomposition based MOEAs (e.g., MOEA/D [39]) adopt similar ideas to obtain a solution set with well balanced convergence and diversity, while the motivation of SDR is completely different. In scalarizing multicriteria approaches and decomposition based MOEAs, solutions are expected to converge to the positions determined by the aggregation functions and weight vectors; by contrast, the proposed SDR adaptively selects the candidate solutions with better convergence and diversity. As a consequence, SDR can adapt to various shapes of Pareto fronts without any aggregation function or weight vector.

\section{EXPERIMENTAL RESULTS}

In this section, we first empirically compare the proposed SDR with eight existing dominance relations on a number of many-objective benchmark problems. Then, a new MOEA, termed NSGA-II/SDR, is established based on SDR and NSGA-II and compared with several stateof-the-art MOEAs tailored for MaOPs. Afterwards, the performance of the proposed SDR is verified on six ex- 
isting MOEAs. Lastly, the effectiveness of the parameter $\bar{\theta}$ in SDR is verified on several test instances. All the experiments are implemented on the PlatEMO ${ }^{1}$ [40].

\section{A. Experimental Settings}

1) Dominance relations: The proposed SDR is compared with Pareto dominance [41], CDAS [6], S-CDAS [24], GPO [7], grid dominance [9], (1-k)-dominance [28], Ldominance [29], and $\theta$-dominance [12] in the experiments. For fairness, the parameter setting for each dominance relation is the same as introduced in Section II-B.

2) Algorithms: To evaluate the performance of NSGAII/SDR, it is compared with four state-of-the-art MOEAs in the experiments, namely, NSGA-III [42], MOEA/DD [18], RVEA [43], and MOMBI-II [44]. The effectiveness of the proposed SDR is also verified on six MOEAs, namely, NSGA-II [2], SPEA2 [20], PESA-II [21], GrEA [9], KnEA [14], and VaEA [15]. In MOEA/DD, the size of neighborhood $T$ is set to $\lceil 0.1 N\rceil$, the neighborhood selection probability $\delta$ is set to 0.9 , and the maximum number of solutions replaced by each offspring $n_{r}$ is set to $\lceil 0.01 N\rceil$, with $N$ denoting the population size. In RVEA, the penalty parameter $\alpha$ in APD is set to 2, and the parameter $f_{r}$ controlling the frequency of reference vector adaption is set to 0.1. In MOMBI-II, the threshold of variance $\alpha$ is set to 0.5 , the tolerance threshold $\epsilon$ is set to 0.001 , and the record size of nadir vectors is set to 5 . In PESA-II, the number of divisions in each objective is set to 6. In GrEA, the number of divisions div is set to 10. In KnEA, the rate of knee points $T$ is set to 0.5.

3) Genetic operators: The simulated binary crossover [45] and polynomial mutation [46] are used to generate offsprings in all the considered MOEAs. The probability of crossover is set to 1 , the probability of mutation is set to $1 / \mathrm{D}$, and the distribution index of them is set to 20 , where $D$ denotes the length of decision variables.

4) Benchmark problems: The widely used DTLZ [47] and WFG [48] test suites are employed as the test problems. Besides, the scaled DTLZ1-DTLZ2 (SDTLZ1-SDTLZ2), convex DTLZ2 (CDTLZ2) [42], inverted DTLZ1 (IDTLZ1) [49], multi-point distance minimization problem (MPDMP), and multi-line distance minimization problem (ML-DMP) [50] are also involved in the experiments. Note that for DTLZ5, the version proposed in [51] is adopted. For all the eleven DTLZ problems, the length of decision variables is set to $K+M-1$, where $M$ denotes the number of objectives and $K$ is set to 5 for DTLZ1, SDTLZ1, and IDTLZ1, 20 for DTLZ7, and 10 for the others. The scaling factor in SDTLZ1 and SDTLZ2 is set to 2. Regarding the nine WFG problems, the length of decision variables is set to $K+L$, where $K$ and $L$ are set to $M-1$ and 10, respectively. For MP-DMP and ML-DMP, the length of decision variables is always 2, and the decision space is set to $[-100,100]^{2}$. Besides, the maximum number of generations is adopted as the

\footnotetext{
${ }^{1}$ http://bimk.ahu.edu.cn/index.php?s=/Index/Software/index.html
}

termination criterion for all the MOEAs, which is set to 200 for all the MOPs.

5) Population size: The population size of all the algorithms is set to the same on the problems with the same number of objectives, namely, 210 for 5-objective problems, 275 for 10-objective problems, and 240 for 15-objective problems. Accordingly, the Das and Dennis's approach with two layers [42] is used to generate the reference vectors used in $\theta$-dominance, NSGA-III, MOEA/DD, RVEA, and MOMBI-II, where the parameters $\left(p_{1}, p_{2}\right)$ controlling the numbers of reference vectors along the outer and inner layers are set to $(6,0),(3,2)$ and $(2,2)$ for 5,10 and 15 objectives, respectively.

6) Performance metric: The inverted generational distance (IGD) [52] and hypervolume (HV) [53] are employed to evaluate the performance of the algorithms, which are the two most widely used performance metric to measure both the convergence and diversity of the obtained non-dominated solution set. A smaller IGD value indicates a better performance of the algorithm, whereas a larger HV value signals a better performance. For calculating IGD, roughly 10000 reference points on the Pareto front of each test instance are sampled by the Das and Dennis's approach with two layers. For calculating $\mathrm{HV}$, the reference point is set to $(1, \ldots, 1)$, and the objective values are normalized by the point $1.1 \times z^{\text {nad }}$ before the calculation, where $z^{\text {nad }}$ denotes the nadir point of the Pareto front. All the algorithms are executed for 30 independent runs on each test instance. Furthermore, the Wilcoxon rank sum test with a significance level of 0.05 is also adopted to analyze the result, where ${ }^{\prime}+{ }^{\prime},{ }^{\prime}-{ }^{\prime}$ and ${ }^{\prime} \approx$ ' indicate that the result is significantly better, significantly worse and statistically similar to that obtained by SDR based algorithms, respectively.

\section{B. Comparing SDR with Other Dominance Relations}

To eliminate the effect of other selection criteria when comparing dominance relations, the proposed SDR is compared with eight existing dominance relations on a simple evolutionary algorithm, where the dominance relation is used as the only selection criterion in the environmental selection. In each generation, $N$ offsprings are generated by genetic operators based on randomly selected parents, with $N$ denoting the population size. Then the population is combined with the offsprings and sorted by a dominance relation, where only the non-dominated candidate solutions survive to the next generation. If the number of non-dominated solutions is larger than $N$, they will be truncated randomly.

The mean IGD values of the nine dominance relation based algorithms on DTLZ1-DTLZ7 are listed in Table I. As can be observed, the SDR based algorithm outperforms the others on 11 out of the 21 test instances, and the $\theta$-dominance, S-CDAS, and GPO achieve 8,1 and 1 best results, respectively.

For visual observations, Fig. 7 plots the parallel coordinates [54] of the non-dominated solution set with 
TABLE I

IGD Values of Nine Dominance Relation Based Evolutionary Algorithms on DTLZ1-DTLZ7 with 5, 10 and 15 Objectives. The Best Result in Each Row is Highlighted.

\begin{tabular}{|c|c|c|c|c|c|c|c|c|c|}
\hline $\operatorname{Problem}(M)$ & $\begin{array}{c}\text { Pareto } \\
\text { dominance }\end{array}$ & CDAS & S-CDAS & GPO & $\begin{array}{c}\text { grid } \\
\text { dominance }\end{array}$ & (1-k)-dominance & L-dominance & $\theta$-dominance & SDR \\
\hline DTLZ1(5) & $2.48 \mathrm{e}-1-$ & $9.66 \mathrm{e}-2-$ & $2.71 \mathrm{e}-1-$ & $1.11 \mathrm{e}-1-$ & $6.72 e+0-$ & $5.79 \mathrm{e}-2-$ & $1.85 \mathrm{e}-1-$ & $5.32 \mathrm{e}-2+$ & $5.55 \mathrm{e}-2$ \\
\hline DTLZ1(10) & $1.75 \mathrm{e}+1-$ & $2.42 \mathrm{e}-1-$ & $1.45 \mathrm{e}-1-$ & $5.32 \mathrm{e}-1-$ & $5.74 \mathrm{e}+0-$ & $2.57 \mathrm{e}-1-$ & $2.32 \mathrm{e}-1-$ & $1.06 \mathrm{e}-1 \approx$ & $1.06 \mathrm{e}-1$ \\
\hline DTLZ1(15) & $1.99 \mathrm{e}+1-$ & $3.63 \mathrm{e}-1-$ & $2.44 \mathrm{e}-1-$ & $7.94 \mathrm{e}-1-$ & $7.60 \mathrm{e}+0-$ & $1.25 \mathrm{e}+0-$ & $3.43 e-1-$ & $1.24 \mathrm{e}-1+$ & $1.34 \mathrm{e}-1$ \\
\hline DTLZ2(10) & $5.99 \mathrm{e}-1-$ & $5.30 \mathrm{e}-1-$ & $1.25 \mathrm{e}+0-$ & $5.07 \mathrm{e}-1-$ & $1.03 e+0-$ & $1.23 e+0-$ & $1.24 \mathrm{e}+0-$ & $4.24 \mathrm{e}-1+$ & $4.71 \mathrm{e}-1$ \\
\hline DTLZ2(15) & $8.63 e-1-$ & $7.48 \mathrm{e}-1-$ & $1.31 \mathrm{e}+0-$ & $7.27 \mathrm{e}-1-$ & $6.17 \mathrm{e}-1+$ & $1.29 \mathrm{e}+0-$ & $1.29 \mathrm{e}+0-$ & $5.28 \mathrm{e}-1+$ & $6.26 \mathrm{e}-1$ \\
\hline DTLZ3(5) & $1.68 \mathrm{e}+0-$ & $6.30 \mathrm{e}-1-$ & $5.32 \mathrm{e}+0-$ & $5.98 \mathrm{e}-1-$ & $4.46 \mathrm{e}+1-$ & $9.40 \mathrm{e}-1-$ & $1.12 \mathrm{e}+0-$ & $2.04 \mathrm{e}+0-$ & $2.40 \mathrm{e}-1$ \\
\hline DTLZ3(10) & $2.84 \mathrm{e}+2-$ & $4.51 \mathrm{e}+0-$ & $3.37 \mathrm{e}+2-$ & $1.41 \mathrm{e}+0-$ & $5.16 e+1-$ & $1.28 \mathrm{e}+0-$ & $1.23 \mathrm{e}+0-$ & $1.73 e+0-$ & $4.75 e-1$ \\
\hline DTLZ4(5) & $2.69 \mathrm{e}-1+$ & $3.44 \mathrm{e}-1 \approx$ & $7.91 \mathrm{e}-1-$ & $3.85 \mathrm{e}-1 \approx$ & $7.92 \mathrm{e}-1-$ & $2.91 \mathrm{e}-1+$ & $9.40 \mathrm{e}-1-$ & $1.65 \mathrm{e}-1+$ & $4.77 \mathrm{e}-1$ \\
\hline DTLZ4(10) & $9.51 \mathrm{e}-1-$ & $6.16 \mathrm{e}-1-$ & $1.24 \mathrm{e}+0-$ & $5.47 \mathrm{e}-1-$ & $8.47 \mathrm{e}-1-$ & $7.93 e-1-$ & $1.15 \mathrm{e}+0-$ & $4.35 \mathrm{e}-1+$ & $5.03 \mathrm{e}-1$ \\
\hline DTLZ4(15) & $1.23 \mathrm{e}+0-$ & 7.30e-1 - & $1.47 \mathrm{e}+0-$ & $7.08 \mathrm{e}-1-$ & $9.60 \mathrm{e}-1-$ & $9.81 \mathrm{e}-1-$ & $1.26 \mathrm{e}+0-$ & $5.45 \mathrm{e}-1+$ & $6.82 \mathrm{e}-1$ \\
\hline DTLZ5(5) & $1.96 \mathrm{e}-2-$ & $1.13 \mathrm{e}-1-$ & $7.42 \mathrm{e}-1-$ & $2.36 \mathrm{e}-1-$ & $7.78 \mathrm{e}-1-$ & $1.64 \mathrm{e}-2-$ & $7.15 \mathrm{e}-1-$ & $7.67 \mathrm{e}-2-$ & $5.55 \mathrm{e}-3$ \\
\hline DTLZ5(10) & $3.11 \mathrm{e}+1-$ & $1.40 \mathrm{e}-1-$ & $3.77 \mathrm{e}+0-$ & $9.86 \mathrm{e}-2-$ & $7.52 \mathrm{e}-1-$ & $6.33 e+0-$ & $7.18 \mathrm{e}-1-$ & $1.05 \mathrm{e}-1-$ & $4.83 e-3$ \\
\hline DTLZ5(15) & $2.80 \mathrm{e}+1-$ & $1.15 \mathrm{e}-1-$ & $1.64 \mathrm{e}+1-$ & $9.75 \mathrm{e}-2-$ & $7.70 \mathrm{e}-1-$ & $1.53 e+1-$ & $7.04 \mathrm{e}-1-$ & $1.36 \mathrm{e}-1-$ & $5.64 \mathrm{e}-3$ \\
\hline DTLZ6(5) & $6.63 e+0-$ & $2.24 \mathrm{e}-1-$ & $9.70 \mathrm{e}+0-$ & $5.45 \mathrm{e}-1-$ & $9.96 \mathrm{e}-1-$ & $1.49 \mathrm{e}-1-$ & $5.17 \mathrm{e}-1-$ & $1.34 \mathrm{e}-1-$ & $7.79 \mathrm{e}-2$ \\
\hline DTLZ6(10) & $6.69 e+0-$ & $2.12 \mathrm{e}-1 \approx$ & $9.95 \mathrm{e}+0-$ & $8.71 \mathrm{e}-1-$ & $1.10 \mathrm{e}+0-$ & $1.04 \mathrm{e}+1-$ & $9.60 \mathrm{e}+0-$ & $2.49 \mathrm{e}-1-$ & $1.25 \mathrm{e}-1$ \\
\hline DTLZ7(10) & $1.54 \mathrm{e}+1-$ & $5.46 \mathrm{e}+0-$ & $1.04 \mathrm{e}+0+$ & $1.13 \mathrm{e}+0+$ & $2.68 \mathrm{e}+0 \approx$ & $4.69 \mathrm{e}+0-$ & $3.39 \mathrm{e}+0-$ & $2.08 \mathrm{e}+0+$ & $2.73 e+0$ \\
\hline DTLZ7(15) & $5.05 e+1-$ & $5.39 \mathrm{e}+0 \approx$ & $3.29 \mathrm{e}+0+$ & $2.08 \mathrm{e}+0+$ & $5.38 \mathrm{e}+0 \approx$ & $1.13 e+1-$ & $3.85 \mathrm{e}+0+$ & $2.84 \mathrm{e}+0+$ & $5.40 \mathrm{e}+0$ \\
\hline$+1-1 \approx$ & $1 / 20 / 0$ & $0 / 18 / 3$ & $2 / 18 / 1$ & $2 / 18 / 1$ & $1 / 18 / 2$ & $1 / 20 / 0$ & $1 / 20 / 0$ & $10 / 10 / 1$ & \\
\hline
\end{tabular}

' $+{ }^{\prime},{ }^{\prime}-{ }^{\prime}$ and ' $\approx$ ' indicate that the result is significantly better, significantly worse and statistically similar to that obtained by SDR based algorithm, respectively.
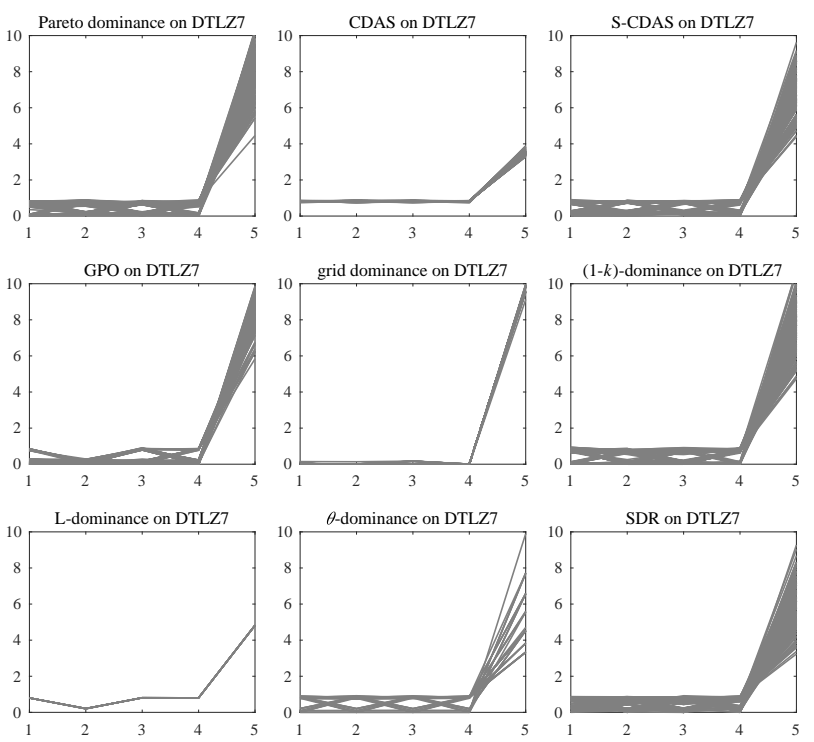

Fig. 7. Parallel coordinates of the non-dominated solution set with the median IGD among 30 runs obtained by nine dominance relation based algorithms on 5-objective DTLZ7.

the median IGD value among 30 runs obtained by the algorithms based on the nine dominance relations on 5-objective DTLZ7, where each polyline in the figure denotes one solution, and each vertex on the polyline denotes one objective value. DTLZ7 has a discontinuous Pareto front challenging the MOEAs in diversity preservation. It can be observed that only Pareto dominance, S-CDAS, (1-k)-dominance, $\theta$-dominance and SDR can make the solutions converge to the whole Pareto front of DTLZ7. Fig. 8 plots the non-dominated solution set with the median IGD value among 30 runs obtained by the
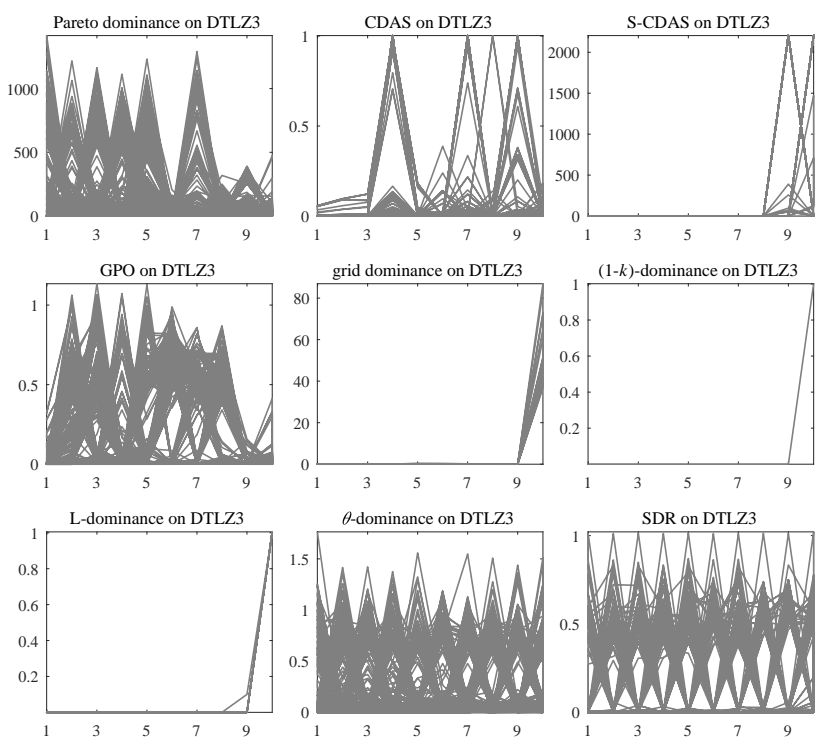

Fig. 8. Parallel coordinates of the non-dominated solution set with the median IGD among 30 runs obtained by nine dominance relation based algorithms on 10-objective DTLZ3.

algorithms based on the nine dominance relations on 10objective DTLZ3. DTLZ3 has a multi-modal landscape, which introduces a large number of local Pareto fronts posing a stiff challenge for MOEAs to converge to the global Pareto front. It can be seen that CDAS, GPO, (1- $k$ dominance, L-dominance, and SDR are able to push the candidate solutions towards the global Pareto front. In terms of diversity, however, only the candidate solutions obtained by SDR show generally proper distributions. Therefore, in conclusion, SDR outperforms existing dominance relations in balancing convergence and diversity. 


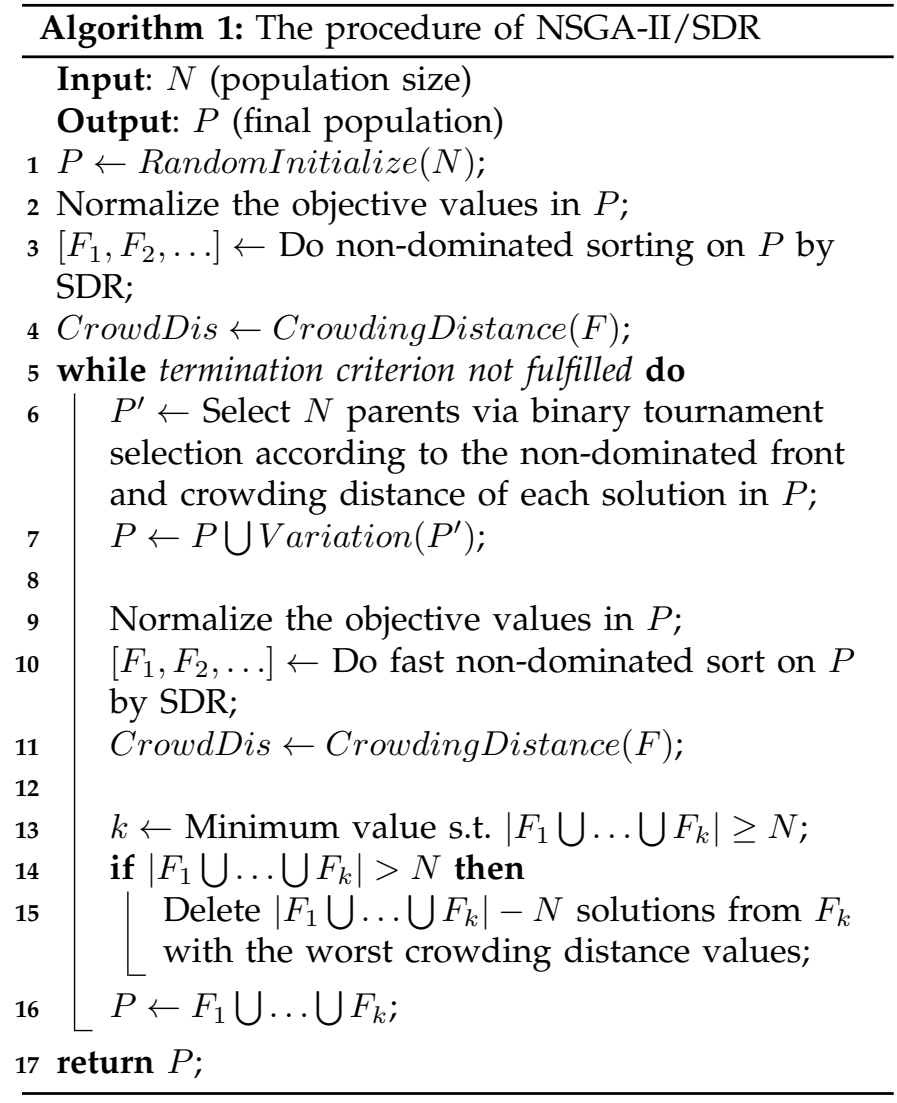

\section{Comparing NSGA-II/SDR with Other MOEAs}

In this subsection, an MOEA, termed NSGA-II/SDR, is developed on the basis of the proposed SDR and NSGAII. As shown by the pseudo code in Algorithm 1, NSGAII/SDR has the same framework with NSGA-II [2], where the main difference is that the Pareto dominance is replaced by the proposed SDR. Besides, the objective values of the candidate solutions are normalized according to the maximum and minimum values in the population before performing SDR in the algorithm. NSGA-II/SDR is compared to four state-of-the-art MOEAs, i.e., NSGAIII [42], MOEA/DD [18], RVEA [43], and MOMBI-II [44]. NSGA-III and MOEA/DD are two algorithms based on both Pareto dominance and decomposition, and they can obtain satisfactory performance on most benchmark MaOPs. RVEA is a recently proposed decomposition based MOEA, which have been verified to be superior over many MOEAs in solving MaOPs. MOMBI-II is an indicator based MOEA, which is also very effective for solving MaOPs.

Table II lists the HV values obtained by the five MOEAs on DTLZ1-DTLZ7, SDTLZ1, SDTLZ2, CDTLZ2, IDTLZ1, WFG1-WFG9, MP-DMP and ML-DMP with 5, 10 and 15 objectives. Among the 66 test instances, NSGAII/SDR have obtained 36 best results, and the numbers of best results obtained by NSGA-III, MOEA/DD, RVEA, and MOMBI-II are 13, 1, 7 and 9, respectively. For further observations, Fig. 9 presents the convergence profiles of IGD values obtained by the five MOEAs

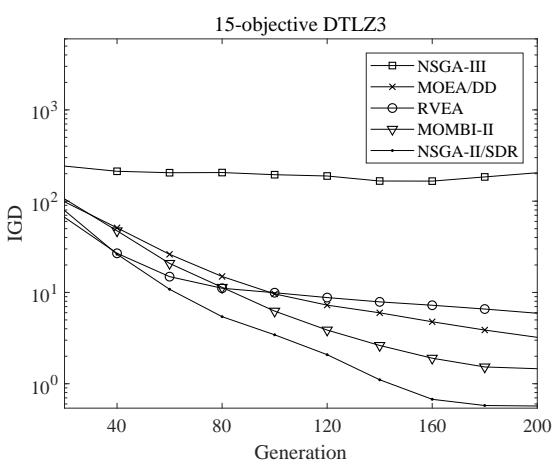

Fig. 9. The convergence profiles of IGD values obtained by NSGAIII, MOEA/DD, RVEA, MOMBI-II, and NSGA-II/SDR on 15-objective DTLZ3, averaged over 30 runs.

on 15-objective DTLZ3, averaged over 30 runs. It is clear that NSGA-II/SDR converges much faster than the other four MOEAs. In addition, Fig. 10 draws the non-dominated solutions obtained by the five MOEAs on 15-objective DTLZ5 and IDTLZ1. For DTLZ5 with a degenerate Pareto front, only the population obtained by NSGA-II/SDR can converge to the Pareto front and spread uniformly. As for IDTLZ1 with an inverted Pareto front, the diversity of the population obtained by NSGAII/SDR is obviously better than the diversity of the populations obtained by the other four MOEAs. Therefore, in conclusion, SDR also outperforms the state-of-the-art MOEAs in balancing convergence and diversity.

\section{Performance of SDR on Existing MOEAs}

The proposed SDR is then embedded in NSGA-II [2], SPEA2 [20], PESA-II [21], GrEA [9], KnEA [14], and VaEA [15], and compared with the original versions of the six MOEAs. NSGA-II, SPEA2, and PESA-II are three classical MOEAs, which have been demonstrated to be effective in solving MOPs. However, their performance deteriorates sharply when meeting MaOPs, since the Pareto dominance relation they used is ineffective on MaOPs. By contrast, GrEA, KnEA, and VaEA are three recently proposed MOEAs combining Pareto dominance with additional selection criteria, which perform fairly well on MaOPs.

For simplicity, the improvement of the MOEAs based on SDR in comparison to their original versions (i.e., those based on Pareto dominance) is recorded. The value in each cell of Table III is calculated as

$$
\text { Improve }=\left(\frac{I G D_{\text {Pareto }}}{I G D_{S D R}}-1\right) \times 100 \%,
$$

where $I G D_{\text {Pareto }}$ and $I G D_{S D R}$ are the mean values of IGD obtained by an MOEA based on Pareto dominance and SDR, respectively. Improve $>0$ indicates that the performance of the MOEA based on SDR is better than the version based on Pareto dominance, and a greater value of Improve indicates a larger improvement of the performance. As shown in Table III, the improvement of SDR based NSGA-II, SPEA2, PESA-II, GrEA, KnEA, and 
TABLE II

HV VALUES OF NSGA-III, MOEA/DD, RVEA, MOMBI-II, AND NSGA-II/SDR ON DTLZ1-DTLZ7, SDTLZ1, SDTLZ2, CDTLZ2, IDTLZ1, WFG1-WFG9, MP-DMP AND ML-DMP with 5, 10 AND 15 OBJECTIVES. THE BEST RESUlt IN EACH ROW IS HighLIGHTED.

\begin{tabular}{|c|c|c|c|c|c|c|}
\hline Problem & $M$ & NSGA-III & MOEA/DD & RVEA & MOMBI-II & NSGA-II/SDR \\
\hline \multirow{3}{*}{ DTLZ1 } & 5 & $9.7578 \mathrm{e}-1(1.30 \mathrm{e}-3)+$ & $9.7737 \mathrm{e}-1(1.11 \mathrm{e}-3)+$ & $9.6109 \mathrm{e}-1(2.14 \mathrm{e}-2) \approx$ & $9.7644 \mathrm{e}-1(7.84 \mathrm{e}-3)+$ & $9.6688 \mathrm{e}-1(2.54 \mathrm{e}-3)$ \\
\hline & 10 & $8.3164 \mathrm{e}-1(2.87 \mathrm{e}-1)-$ & $9.9447 \mathrm{e}-1(2.15 \mathrm{e}-3)-$ & $9.9603 \mathrm{e}-1(1.58 \mathrm{e}-3) \approx$ & $9.2316 \mathrm{e}-1(7.23 \mathrm{e}-2)-$ & $9.9611 \mathrm{e}-1(4.82 \mathrm{e}-4)$ \\
\hline & 15 & $5.4543 \mathrm{e}-1(4.27 \mathrm{e}-1)-$ & $9.4016 \mathrm{e}-1(1.21 \mathrm{e}-2)-$ & $9.8947 \mathrm{e}-1(5.39 \mathrm{e}-3)-$ & $8.6019 \mathrm{e}-1(9.52 \mathrm{e}-2)-$ & $9.9750 \mathrm{e}-1(6.38 \mathrm{e}-4)$ \\
\hline \multirow{3}{*}{ DTLZ2 } & 5 & $8.0899 \mathrm{e}-1(6.26 \mathrm{e}-4)+$ & $8.1031 \mathrm{e}-1(6.46 \mathrm{e}-4)+$ & $8.1046 \mathrm{e}-1(6.53 \mathrm{e}-4)+$ & $8.0998 \mathrm{e}-1(4.60 \mathrm{e}-4)+$ & $7.9283 e-1(2.10 e-3)$ \\
\hline & 10 & $9.3703 \mathrm{e}-1(5.03 \mathrm{e}-2)-$ & $9.6697 \mathrm{e}-1(4.63 \mathrm{e}-4)+$ & $9.6756 \mathrm{e}-1(3.05 \mathrm{e}-4)+$ & $9.6558 \mathrm{e}-1(3.04 \mathrm{e}-3)+$ & $9.6236 \mathrm{e}-1(2.06 \mathrm{e}-3)$ \\
\hline & 15 & $9.0718 \mathrm{e}-1(5.97 \mathrm{e}-2)-$ & $8.7942 \mathrm{e}-1(2.04 \mathrm{e}-2)-$ & $9.7906 \mathrm{e}-1(7.07 \mathrm{e}-3)-$ & $8.9199 \mathrm{e}-1(4.96 \mathrm{e}-2)-$ & $9.8999 \mathrm{e}-1(1.36 \mathrm{e}-3)$ \\
\hline \multirow{3}{*}{ DTLZ3 } & 5 & $2.3919 \mathrm{e}-2(9.88 \mathrm{e}-2)-$ & $1.5554 \mathrm{e}-2(4.74 \mathrm{e}-2)-$ & $2.4240 \mathrm{e}-2(6.66 \mathrm{e}-2)-$ & $6.6809 \mathrm{e}-1(1.70 \mathrm{e}-1) \approx$ & $6.8342 \mathrm{e}-1(1.91 \mathrm{e}-1)$ \\
\hline & 10 & $0.0000 \mathrm{e}+0(0.00 \mathrm{e}+0)-$ & $8.9910 \mathrm{e}-2(2.22 \mathrm{e}-1)-$ & $8.3030 \mathrm{e}-3(2.51 \mathrm{e}-2)-$ & $7.4329 \mathrm{e}-1(2.08 \mathrm{e}-1)-$ & $7.6288 \mathrm{e}-1(3.91 \mathrm{e}-1)$ \\
\hline & 15 & $0.0000 \mathrm{e}+0(0.00 \mathrm{e}+0)-$ & $2.6049 \mathrm{e}-1(3.39 \mathrm{e}-1)-$ & $1.6921 \mathrm{e}-1(3.18 \mathrm{e}-1)-$ & $3.2682 \mathrm{e}-1(1.34 \mathrm{e}-1)-$ & $8.3682 \mathrm{e}-1(3.46 \mathrm{e}-1)$ \\
\hline \multirow{3}{*}{ DTLZ4 } & 5 & $8.0327 \mathrm{e}-1(2.34 \mathrm{e}-2)+$ & $8.1065 \mathrm{e}-1(8.02 \mathrm{e}-4)+$ & $8.1079 \mathrm{e}-1(4.59 \mathrm{e}-4)+$ & $8.0479 \mathrm{e}-1(2.16 \mathrm{e}-2)+$ & $7.9241 \mathrm{e}-1(2.60 \mathrm{e}-3)$ \\
\hline & 10 & $9.5517 \mathrm{e}-1(2.37 \mathrm{e}-2)-$ & $9.6900 \mathrm{e}-1(3.18 \mathrm{e}-4)+$ & $9.6978 \mathrm{e}-1(3.43 \mathrm{e}-4)+$ & $9.7332 \mathrm{e}-1(2.37 \mathrm{e}-4)+$ & $9.6017 e-1(2.36 e-3)$ \\
\hline & 15 & $9.5763 \mathrm{e}-1(4.43 \mathrm{e}-2) \approx$ & $9.8801 \mathrm{e}-1(1.04 \mathrm{e}-3) \approx$ & $9.9074 \mathrm{e}-1(1.45 \mathrm{e}-3)+$ & $9.8889 \mathrm{e}-1(2.61 \mathrm{e}-3) \approx$ & $9.8797 \mathrm{e}-1(1.38 \mathrm{e}-3)$ \\
\hline \multirow{3}{*}{ DTLZ5 } & 5 & $2.6840 \mathrm{e}-1(6.98 \mathrm{e}-3) \approx$ & $2.3627 \mathrm{e}-1(5.62 \mathrm{e}-3)-$ & $2.4207 e-1(7.15 e-3)-$ & $2.2197 \mathrm{e}-1(1.16 \mathrm{e}-2)$ & $2.7303 e-1(5.83 e-4)$ \\
\hline & 10 & $3.4597 \mathrm{e}-3(8.90 \mathrm{e}-3)-$ & $1.9064 \mathrm{e}-1(1.35 \mathrm{e}-2)-$ & $1.8683 \mathrm{e}-1(1.10 \mathrm{e}-2)-$ & $9.9535 \mathrm{e}-2(1.32 \mathrm{e}-2)-$ & $2.3611 \mathrm{e}-1(5.66 \mathrm{e}-4)$ \\
\hline & 15 & $3.9348 \mathrm{e}-4(1.76 \mathrm{e}-3)-$ & $1.8255 \mathrm{e}-1(4.20 \mathrm{e}-3)-$ & $1.4097 \mathrm{e}-1(3.67 \mathrm{e}-2)-$ & $9.5089 \mathrm{e}-2(6.26 \mathrm{e}-3)-$ & $2.3034 \mathrm{e}-1(6.19 \mathrm{e}-4)$ \\
\hline \multirow{3}{*}{ DTLZ6 } & 5 & $1.4621 \mathrm{e}-1(5.34 \mathrm{e}-2)-$ & $2.2614 \mathrm{e}-1(8.10 \mathrm{e}-3)-$ & $1.8357 \mathrm{e}-1(3.00 \mathrm{e}-2)-$ & $1.6779 \mathrm{e}-1(3.42 \mathrm{e}-4)-$ & $2.4552 \mathrm{e}-1(5.17 \mathrm{e}-3)$ \\
\hline & 10 & $0.0000 \mathrm{e}+0(0.00 \mathrm{e}+0)-$ & $1.7046 \mathrm{e}-1(4.21 \mathrm{e}-2)-$ & $1.2152 \mathrm{e}-1(3.47 \mathrm{e}-2)-$ & $1.0793 \mathrm{e}-1(2.34 \mathrm{e}-2)-$ & $1.9054 \mathrm{e}-1(8.13 \mathrm{e}-3)$ \\
\hline & 15 & $0.0000 \mathrm{e}+0(0.00 \mathrm{e}+0)-$ & $1.0226 \mathrm{e}-1(7.04 \mathrm{e}-2)-$ & $8.8366 \mathrm{e}-2(4.24 \mathrm{e}-2)-$ & $9.4380 \mathrm{e}-2(1.04 \mathrm{e}-2)-$ & $1.8689 \mathrm{e}-1(7.33 \mathrm{e}-3)$ \\
\hline \multirow{3}{*}{ DTLZ7 } & 5 & $2.4248 \mathrm{e}-1(4.36 \mathrm{e}-3)+$ & $1.0474 \mathrm{e}-1(2.89 \mathrm{e}-2)-$ & $2.2050 \mathrm{e}-1(3.81 \mathrm{e}-3) \approx$ & $2.5430 \mathrm{e}-1(9.73 \mathrm{e}-3)+$ & $2.1727 \mathrm{e}-1(1.22 \mathrm{e}-2)$ \\
\hline & 10 & $1.1461 \mathrm{e}-1(1.12 \mathrm{e}-2)+$ & $7.9968 \mathrm{e}-5(3.37 \mathrm{e}-5)-$ & $1.2376 \mathrm{e}-1(1.96 \mathrm{e}-2)+$ & $1.5628 \mathrm{e}-1(9.34 \mathrm{e}-3)+$ & $6.4970 \mathrm{e}-4(4.05 \mathrm{e}-4)$ \\
\hline & 15 & $4.6537 \mathrm{e}-1(2.89 \mathrm{e}-2)+$ & $9.5239 \mathrm{e}-3(1.30 \mathrm{e}-3)-$ & $2.2530 \mathrm{e}-1(6.42 \mathrm{e}-2)+$ & $5.5850 \mathrm{e}-1(4.82 \mathrm{e}-4)+$ & $2.2383 \mathrm{e}-2(1.39 \mathrm{e}-3)$ \\
\hline \multirow{3}{*}{ SDTLZ1 } & 5 & $9.7967 \mathrm{e}-1(2.02 \mathrm{e}-4)+$ & $9.0148 \mathrm{e}-1(3.71 \mathrm{e}-3)-$ & $9.7922 \mathrm{e}-1(8.65 \mathrm{e}-4)+$ & $9.7949 \mathrm{e}-1(2.15 \mathrm{e}-4)+$ & $9.4138 \mathrm{e}-1(6.46 \mathrm{e}-3)$ \\
\hline & 10 & $9.9260 \mathrm{e}-1(2.01 \mathrm{e}-2)+$ & $7.9979 \mathrm{e}-1(1.85 \mathrm{e}-2)-$ & $9.1879 \mathrm{e}-1(3.82 \mathrm{e}-2)-$ & $9.3820 \mathrm{e}-1(4.01 \mathrm{e}-2) \approx$ & $9.5702 \mathrm{e}-1(1.80 \mathrm{e}-2)$ \\
\hline & 15 & $9.6476 \mathrm{e}-1(1.24 \mathrm{e}-1)+$ & $7.9242 \mathrm{e}-1(3.25 \mathrm{e}-2) \approx$ & $8.0607 \mathrm{e}-1(5.62 \mathrm{e}-2) \approx$ & $8.5317 \mathrm{e}-1(7.47 \mathrm{e}-2)+$ & $7.0406 \mathrm{e}-1(2.54 \mathrm{e}-1)$ \\
\hline \multirow{3}{*}{ SDTLZ2 } & 5 & $8.1242 \mathrm{e}-1(3.88 \mathrm{e}-4)+$ & $6.7446 \mathrm{e}-1(4.88 \mathrm{e}-3)-$ & $8.1160 \mathrm{e}-1(3.78 \mathrm{e}-4)+$ & $8.1200 \mathrm{e}-1(3.41 \mathrm{e}-4)+$ & $7.9412 \mathrm{e}-1(2.27 \mathrm{e}-3)$ \\
\hline & 10 & $9.6316 \mathrm{e}-1(9.08 \mathrm{e}-3)-$ & $4.9585 \mathrm{e}-1(2.29 \mathrm{e}-2)-$ & $8.2447 \mathrm{e}-1(3.07 \mathrm{e}-2)-$ & $9.7055 \mathrm{e}-1(2.42 \mathrm{e}-4)+$ & $9.6437 \mathrm{e}-1(2.60 \mathrm{e}-3)$ \\
\hline & 15 & $9.7634 \mathrm{e}-1(1.00 \mathrm{e}-2)+$ & $4.6178 \mathrm{e}-1(6.61 \mathrm{e}-2)-$ & 6.3847e-1 (9.54e-2) - & $6.1964 \mathrm{e}-1(1.30 \mathrm{e}-1)-$ & $8.6036 \mathrm{e}-1(5.79 \mathrm{e}-2)$ \\
\hline \multirow{3}{*}{ CDTLZ2 } & 5 & $9.9939 \mathrm{e}-1(3.16 \mathrm{e}-5)+$ & $9.9257 \mathrm{e}-1(1.30 \mathrm{e}-3)+$ & $9.9773 \mathrm{e}-1(5.11 \mathrm{e}-4)+$ & $9.9930 \mathrm{e}-1(3.58 \mathrm{e}-5)+$ & $9.8160 \mathrm{e}-1(4.30 \mathrm{e}-3)$ \\
\hline & 10 & $9.9972 \mathrm{e}-1(4.87 \mathrm{e}-4)+$ & $9.7719 \mathrm{e}-1(2.19 \mathrm{e}-3)-$ & $9.9543 \mathrm{e}-1(1.26 \mathrm{e}-3) \approx$ & $1.0000 \mathrm{e}+0(9.99 \mathrm{e}-7)+$ & $9.9452 \mathrm{e}-1(1.67 \mathrm{e}-3)$ \\
\hline & 15 & $9.9985 \mathrm{e}-1(2.20 \mathrm{e}-4)+$ & $9.9405 \mathrm{e}-1(1.03 \mathrm{e}-3)-$ & $9.9461 \mathrm{e}-1(1.46 \mathrm{e}-3)-$ & $9.9917 \mathrm{e}-1(1.15 \mathrm{e}-3)+$ & $9.9604 \mathrm{e}-1(7.90 \mathrm{e}-4)$ \\
\hline \multirow{3}{*}{ IDTLZ1 } & 5 & $8.1792 \mathrm{e}-3(4.03 \mathrm{e}-4)-$ & $5.9753 \mathrm{e}-3(5.03 \mathrm{e}-4)-$ & $2.7461 \mathrm{e}-3(8.00 \mathrm{e}-4)-$ & $5.4801 \mathrm{e}-3(3.92 \mathrm{e}-4)-$ & $1.3043 \mathrm{e}-2(2.06 \mathrm{e}-4)$ \\
\hline & 10 & $4.8550 \mathrm{e}-7(9.39 \mathrm{e}-8)-$ & $4.4125 \mathrm{e}-8(1.41 \mathrm{e}-8)-$ & $1.2406 \mathrm{e}-8(5.63 \mathrm{e}-9)-$ & $1.4913 \mathrm{e}-7(2.74 \mathrm{e}-8)-$ & $6.2035 \mathrm{e}-7(3.26 \mathrm{e}-7)$ \\
\hline & 15 & $2.1250 \mathrm{e}-5(2.40 \mathrm{e}-6)-$ & $7.5419 \mathrm{e}-6(8.03 \mathrm{e}-7)-$ & $4.6044 \mathrm{e}-6(5.00 \mathrm{e}-7)-$ & $7.3176 \mathrm{e}-6(1.94 \mathrm{e}-7)-$ & $6.4529 e-5(1.02 e-5)$ \\
\hline \multirow{3}{*}{ WFG1 } & 5 & $5.4887 \mathrm{e}-1(4.08 \mathrm{e}-2)-$ & $4.6747 \mathrm{e}-1(5.87 \mathrm{e}-2)-$ & $6.0114 \mathrm{e}-1(4.80 \mathrm{e}-2)-$ & $9.8261 \mathrm{e}-1(9.75 \mathrm{e}-3)+$ & $7.5060 \mathrm{e}-1(3.58 \mathrm{e}-2)$ \\
\hline & 10 & $4.2657 \mathrm{e}-1(4.11 \mathrm{e}-2)-$ & $4.8213 \mathrm{e}-1(5.78 \mathrm{e}-2)-$ & $6.0616 \mathrm{e}-1(7.32 \mathrm{e}-2)-$ & $9.9741 \mathrm{e}-1(5.41 \mathrm{e}-4)+$ & $7.8961 \mathrm{e}-1(4.25 \mathrm{e}-2)$ \\
\hline & 15 & $4.3844 \mathrm{e}-1(7.47 \mathrm{e}-2)-$ & $3.4950 \mathrm{e}-1(4.44 \mathrm{e}-2)-$ & $6.5608 \mathrm{e}-1(8.82 \mathrm{e}-2)-$ & $9.8789 \mathrm{e}-1(2.86 \mathrm{e}-2)+$ & $8.9124 \mathrm{e}-1(6.19 \mathrm{e}-2)$ \\
\hline & 5 & $9.8242 \mathrm{e}-1(3.30 \mathrm{e}-3)+$ & $9.5998 \mathrm{e}-1(4.55 \mathrm{e}-3)-$ & $9.7596 \mathrm{e}-1(3.24 \mathrm{e}-3)+$ & $9.7462 \mathrm{e}-1(1.34 \mathrm{e}-2)+$ & $9.6927 e-1(6.22 e-3)$ \\
\hline WFG2 & 10 & $9.9340 \mathrm{e}-1(4.29 \mathrm{e}-3)+$ & $9.6365 \mathrm{e}-1(8.19 \mathrm{e}-3)-$ & $9.7083 \mathrm{e}-1(5.61 \mathrm{e}-3)-$ & $9.8646 \mathrm{e}-1(2.24 \mathrm{e}-2)+$ & $9.7874 \mathrm{e}-1(2.24 \mathrm{e}-2)$ \\
\hline & 15 & $9.8798 \mathrm{e}-1(4.73 \mathrm{e}-3)+$ & $9.6064 \mathrm{e}-1(2.04 \mathrm{e}-2) \approx$ & $9.5399 \mathrm{e}-1(3.25 \mathrm{e}-2) \approx$ & $8.9100 \mathrm{e}-1(7.56 \mathrm{e}-2)-$ & $9.3641 \mathrm{e}-1(8.70 \mathrm{e}-2)$ \\
\hline & 5 & $6.3239 \mathrm{e}-1(6.77 \mathrm{e}-3) \approx$ & $5.9868 \mathrm{e}-1(1.13 \mathrm{e}-2)-$ & $6.1298 \mathrm{e}-1(1.65 \mathrm{e}-2)-$ & $5.3767 \mathrm{e}-1(1.53 \mathrm{e}-2)-$ & $6.3697 e-1(8.75 e-3)$ \\
\hline WFG3 & 10 & $6.4862 \mathrm{e}-1(3.14 \mathrm{e}-2)+$ & $4.9850 \mathrm{e}-1(1.31 \mathrm{e}-2)-$ & $2.7357 \mathrm{e}-1(7.37 \mathrm{e}-2)-$ & $1.9808 \mathrm{e}-1(4.47 \mathrm{e}-3)-$ & $6.2874 \mathrm{e}-1(1.78 \mathrm{e}-2)$ \\
\hline & 15 & $5.8129 \mathrm{e}-1(3.93 \mathrm{e}-2)-$ & $2.6392 \mathrm{e}-1(2.09 \mathrm{e}-2)-$ & $2.0569 \mathrm{e}-1(2.05 \mathrm{e}-2)-$ & $1.7051 \mathrm{e}-1(4.99 \mathrm{e}-3)-$ & $6.6286 \mathrm{e}-1(3.17 \mathrm{e}-2)$ \\
\hline & 5 & $7.7513 \mathrm{e}-1(2.67 \mathrm{e}-3) \approx$ & $7.5530 \mathrm{e}-1(2.65 \mathrm{e}-3)-$ & $7.7691 \mathrm{e}-1(2.74 \mathrm{e}-3) \approx$ & $7.0241 \mathrm{e}-1(5.55 \mathrm{e}-2)-$ & $7.7482 \mathrm{e}-1(4.62 \mathrm{e}-3)$ \\
\hline WFG4 & 10 & $9.0350 \mathrm{e}-1(6.87 \mathrm{e}-3)-$ & $7.7140 \mathrm{e}-1(1.95 \mathrm{e}-2)-$ & $8.8229 \mathrm{e}-1(9.50 \mathrm{e}-3)-$ & $8.8357 \mathrm{e}-1(3.29 \mathrm{e}-2)-$ & $9.3383 e-1(5.47 e-3)$ \\
\hline & 15 & $8.2530 \mathrm{e}-1(4.03 \mathrm{e}-2)-$ & $7.3239 \mathrm{e}-1(3.53 \mathrm{e}-2)-$ & $8.7665 \mathrm{e}-1(2.09 \mathrm{e}-2)-$ & $6.2027 \mathrm{e}-1(9.32 \mathrm{e}-2)-$ & $9.4964 \mathrm{e}-1(7.56 \mathrm{e}-3)$ \\
\hline & 5 & $7.4711 \mathrm{e}-1(2.42 \mathrm{e}-3)+$ & $7.2998 \mathrm{e}-1(5.11 \mathrm{e}-2)-$ & $7.4683 \mathrm{e}-1(2.05 \mathrm{e}-3)+$ & $7.0329 \mathrm{e}-1(1.92 \mathrm{e}-2)-$ & $7.4040 \mathrm{e}-1(3.25 \mathrm{e}-3)$ \\
\hline WFG5 & 10 & $8.7105 \mathrm{e}-1(3.36 \mathrm{e}-3)-$ & $7.1420 \mathrm{e}-1(1.65 \mathrm{e}-2)-$ & $8.6404 \mathrm{e}-1(4.80 \mathrm{e}-3)-$ & $7.9123 \mathrm{e}-1(6.60 \mathrm{e}-3)-$ & $8.8845 e-1(2.67 e-3)$ \\
\hline & 15 & $8.1199 \mathrm{e}-1(3.04 \mathrm{e}-2) \approx$ & $7.0167 \mathrm{e}-1(2.80 \mathrm{e}-2)-$ & $8.5023 \mathrm{e}-1(9.27 \mathrm{e}-3) \approx$ & $3.0148 \mathrm{e}-1(7.59 \mathrm{e}-2)-$ & $7.8236 \mathrm{e}-1(1.15 \mathrm{e}-1)$ \\
\hline & 5 & $7.1818 \mathrm{e}-1(1.53 \mathrm{e}-2) \approx$ & $6.9347 \mathrm{e}-1(1.57 \mathrm{e}-2)-$ & $7.2154 \mathrm{e}-1(1.22 \mathrm{e}-2) \approx$ & $6.4927 \mathrm{e}-1(4.37 \mathrm{e}-2)-$ & 7.1859e-1 (1.26e-2) \\
\hline WFG6 & 10 & $8.4510 \mathrm{e}-1(1.87 \mathrm{e}-2)-$ & $7.1767 \mathrm{e}-1(2.05 \mathrm{e}-2)-$ & $8.0343 \mathrm{e}-1(2.48 \mathrm{e}-2)-$ & $7.7126 \mathrm{e}-1(2.21 \mathrm{e}-2)-$ & $8.7678 \mathrm{e}-1(1.43 \mathrm{e}-2)$ \\
\hline & 15 & $7.5781 \mathrm{e}-1(2.73 \mathrm{e}-2)-$ & $6.3532 \mathrm{e}-1(3.55 \mathrm{e}-2)-$ & $7.6722 \mathrm{e}-1(4.79 \mathrm{e}-2)-$ & $6.3532 \mathrm{e}-1(6.79 \mathrm{e}-2)-$ & $8.8247 \mathrm{e}-1(2.02 \mathrm{e}-2)$ \\
\hline & 5 & $7.8409 \mathrm{e}-1(3.18 \mathrm{e}-3)-$ & $7.5887 \mathrm{e}-1(5.10 \mathrm{e}-3)-$ & $7.8783 \mathrm{e}-1(2.87 \mathrm{e}-3)-$ & $7.6818 \mathrm{e}-1(2.19 \mathrm{e}-2)-$ & $7.9003 e-1(2.29 e-3)$ \\
\hline WFG7 & 10 & $9.2322 \mathrm{e}-1(1.17 \mathrm{e}-2)-$ & $8.2767 \mathrm{e}-1(1.69 \mathrm{e}-2)-$ & $8.9959 \mathrm{e}-1(7.75 \mathrm{e}-3)-$ & $8.9003 e-1(5.67 e-3)-$ & $9.5554 \mathrm{e}-1(2.23 \mathrm{e}-3)$ \\
\hline & 15 & $8.2190 \mathrm{e}-1(4.34 \mathrm{e}-2)-$ & $5.0136 \mathrm{e}-1(4.95 \mathrm{e}-2)-$ & $7.6203 \mathrm{e}-1(1.01 \mathrm{e}-1)-$ & $7.8296 \mathrm{e}-1(9.88 \mathrm{e}-2)-$ & $9.5943 e-1(6.86 e-3)$ \\
\hline & 5 & $6.6566 \mathrm{e}-1(4.96 \mathrm{e}-3) \approx$ & $6.4639 \mathrm{e}-1(1.22 \mathrm{e}-2)-$ & $6.6388 \mathrm{e}-1(5.53 \mathrm{e}-3)-$ & $3.6935 \mathrm{e}-1(1.37 \mathrm{e}-2)-$ & $6.6724 \mathrm{e}-1(4.22 \mathrm{e}-3)$ \\
\hline WFG8 & 10 & $7.8899 \mathrm{e}-1(3.06 \mathrm{e}-2)-$ & $7.0532 \mathrm{e}-1(5.24 \mathrm{e}-2)-$ & $7.1108 \mathrm{e}-1(9.90 \mathrm{e}-2)-$ & $6.6086 \mathrm{e}-1(4.51 \mathrm{e}-2)-$ & $8.7806 \mathrm{e}-1(2.47 \mathrm{e}-2)$ \\
\hline & 15 & $6.5570 \mathrm{e}-1(1.53 \mathrm{e}-1)-$ & $4.9298 \mathrm{e}-1(5.35 \mathrm{e}-2)-$ & $6.8953 \mathrm{e}-1(1.07 \mathrm{e}-1)-$ & $3.2596 \mathrm{e}-1(8.16 \mathrm{e}-2)-$ & $8.5400 \mathrm{e}-1(1.11 \mathrm{e}-2)$ \\
\hline & 5 & $7.2308 \mathrm{e}-1(8.87 \mathrm{e}-3)-$ & $6.9055 \mathrm{e}-1(1.47 \mathrm{e}-2)-$ & $7.2710 \mathrm{e}-1(5.15 \mathrm{e}-3)-$ & $4.7639 \mathrm{e}-1(4.16 \mathrm{e}-2)-$ & $7.5551 \mathrm{e}-1(4.12 \mathrm{e}-3)$ \\
\hline WFG9 & 10 & $8.2432 \mathrm{e}-1(3.77 \mathrm{e}-2)-$ & $6.0699 \mathrm{e}-1(3.73 \mathrm{e}-2)-$ & $7.9557 \mathrm{e}-1(2.45 \mathrm{e}-2)-$ & $8.0764 \mathrm{e}-1(1.22 \mathrm{e}-2)-$ & $8.9294 \mathrm{e}-1(4.02 \mathrm{e}-3)$ \\
\hline & 15 & $7.8714 \mathrm{e}-1(6.58 \mathrm{e}-2)-$ & $6.0814 \mathrm{e}-1(4.45 \mathrm{e}-2)-$ & $7.3791 \mathrm{e}-1(4.89 \mathrm{e}-2)-$ & $2.8607 \mathrm{e}-1(7.35 \mathrm{e}-2)-$ & $8.7049 \mathrm{e}-1(1.94 \mathrm{e}-2)$ \\
\hline & 5 & $1.1467 \mathrm{e}-1(1.71 \mathrm{e}-3)-$ & $8.1875 \mathrm{e}-2(6.26 \mathrm{e}-3)-$ & $7.5149 \mathrm{e}-2(4.42 \mathrm{e}-3)-$ & $8.2525 \mathrm{e}-2(3.82 \mathrm{e}-3)-$ & $1.2441 \mathrm{e}-1(4.13 \mathrm{e}-4)$ \\
\hline MP-DMP & 10 & $9.9498 \mathrm{e}-3(1.67 \mathrm{e}-4)-$ & $6.0229 \mathrm{e}-3(2.70 \mathrm{e}-4)-$ & $4.9983 \mathrm{e}-3(4.55 \mathrm{e}-4)-$ & $2.1744 \mathrm{e}-3(3.85 \mathrm{e}-5)-$ & $1.0881 \mathrm{e}-2(1.04 \mathrm{e}-4)$ \\
\hline & 15 & $5.4250 \mathrm{e}-4(2.11 \mathrm{e}-5)-$ & $3.2617 \mathrm{e}-4(2.60 \mathrm{e}-5)-$ & $2.3297 \mathrm{e}-4(3.26 \mathrm{e}-5)-$ & $9.7809 \mathrm{e}-5(2.37 \mathrm{e}-6)-$ & $6.2072 \mathrm{e}-4(1.81 \mathrm{e}-5)$ \\
\hline & 5 & $2.9608 \mathrm{e}-1(5.15 \mathrm{e}-3)+$ & $2.5273 \mathrm{e}-1(9.71 \mathrm{e}-4)-$ & $2.4096 \mathrm{e}-1(5.53 \mathrm{e}-3)-$ & $2.3047 \mathrm{e}-1(1.73 \mathrm{e}-2)-$ & $2.9013 \mathrm{e}-1(2.75 \mathrm{e}-3)$ \\
\hline ML-DMP & 10 & $1.2122 \mathrm{e}-2(1.62 \mathrm{e}-3)-$ & $7.8785 \mathrm{e}-3(9.44 \mathrm{e}-5)-$ & $5.2727 \mathrm{e}-3(6.11 \mathrm{e}-4)-$ & $2.2232 \mathrm{e}-3(7.08 \mathrm{e}-5)-$ & $1.6035 e-2(1.99 e-4)$ \\
\hline & 15 & $9.5381 \mathrm{e}-4(8.71 \mathrm{e}-5)-$ & $6.0735 \mathrm{e}-4(4.93 \mathrm{e}-5)-$ & $3.3211 \mathrm{e}-4(6.63 \mathrm{e}-5)-$ & $1.1544 \mathrm{e}-4(8.46 \mathrm{e}-6)-$ & $1.0989 \mathrm{e}-3(3.33 \mathrm{e}-5)$ \\
\hline
\end{tabular}

' + ',$'-{ }^{\prime}$ and ' $\approx$ ' indicate that the result is significantly better, significantly worse and statistically similar to that obtained by NSGA-II/SDR, respectively. 

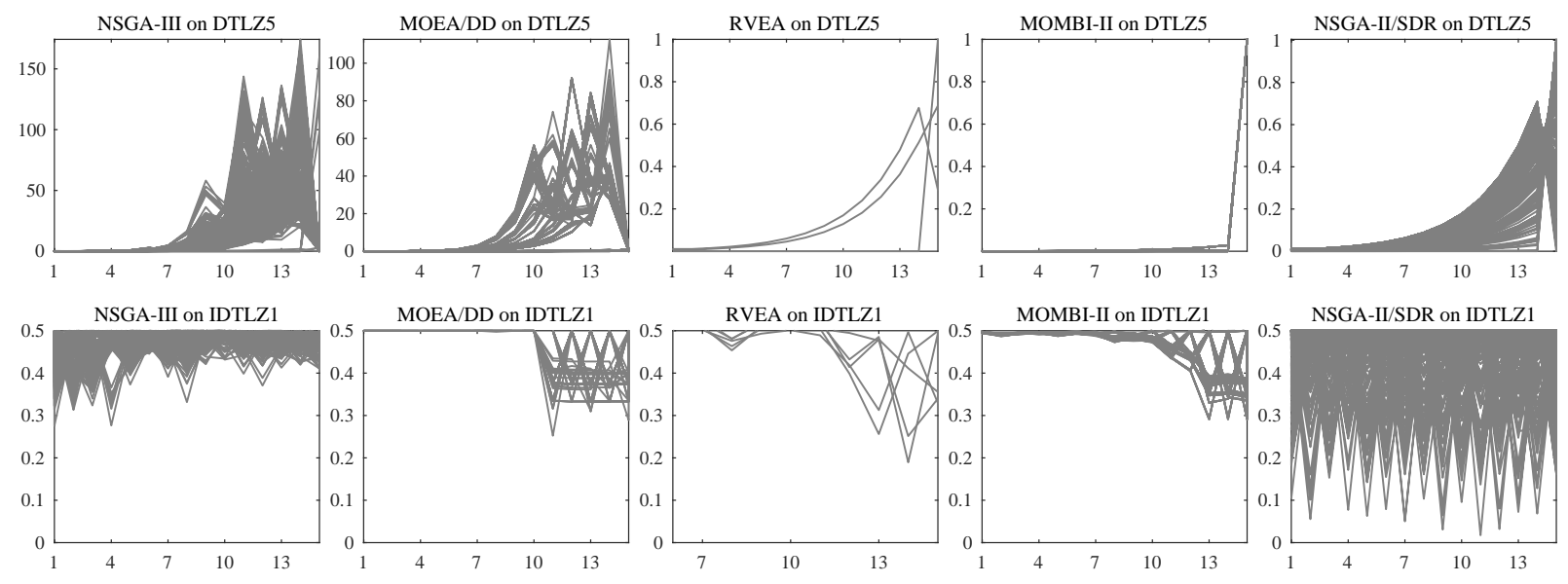

Fig. 10. Parallel coordinates of the non-dominated solution set with the median HV among 30 runs obtained by NSGA-III, MOEA/DD, RVEA, MOMBI-II, and NSGA-II/SDR on DTLZ5 and IDTLZ1 with 15 objectives.

TABLE III

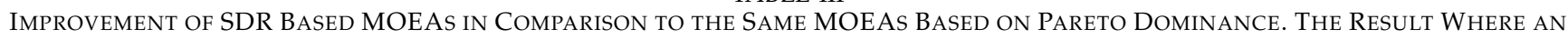
MOEA BASED ON SDR is BETTER THAN IT BASED ON PARETO DOMINANCE IS Highlighted.

\begin{tabular}{|c|c|c|c|c|c|c|}
\hline Problem( $M$ ) & NSGA-II & SPEA2 & PESA-II & GrEA & KnEA & VaEA \\
\hline DTLZ1(5) & $246.95 \%$ & $19.66 \%$ & $131748.00 \%$ & $214.50 \%$ & $230.09 \%$ & $101.95 \%$ \\
DTLZ1(10) & $11817.36 \%$ & $142907.07 \%$ & $54167.23 \%$ & $250.88 \%$ & $4639.74 \%$ & $101.25 \%$ \\
DTLZ1(15) & $10313.99 \%$ & $182674.26 \%$ & $32315.21 \%$ & $198.35 \%$ & $2886.15 \%$ & $235.76 \%$ \\
\hline DTLZ2(5) & $17.79 \%$ & $6.04 \%$ & $10.18 \%$ & $-6.44 \%$ & $-3.43 \%$ & $-4.19 \%$ \\
DTLZ2(10) & $253.63 \%$ & $433.91 \%$ & $29.87 \%$ & $-6.50 \%$ & $-1.35 \%$ & $3.15 \%$ \\
DTLZ2(15) & $150.29 \%$ & $349.11 \%$ & $60.08 \%$ & $-7.93 \%$ & $-4.61 \%$ & $11.71 \%$ \\
\hline DTLZ3(5) & $349.16 \%$ & $10.39 \%$ & $116928.10 \%$ & $292.97 \%$ & $135.62 \%$ & $85.16 \%$ \\
DTLZ3(10) & $235168.06 \%$ & $327702.21 \%$ & $83404.53 \%$ & $735.71 \%$ & $62313.46 \%$ & $2924.73 \%$ \\
DTLZ3(15) & $187636.42 \%$ & $302265.42 \%$ & $66003.62 \%$ & $33096.54 \%$ & $85689.74 \%$ & $3744.82 \%$ \\
\hline DTLZ4(5) & $14.01 \%$ & $35.21 \%$ & $-26.28 \%$ & $-4.91 \%$ & $-3.46 \%$ & $-3.52 \%$ \\
DTLZ4(10) & $249.16 \%$ & $421.45 \%$ & $86.94 \%$ & $-6.28 \%$ & $-0.43 \%$ & $6.79 \%$ \\
DTLZ4(15) & $167.30 \%$ & $345.25 \%$ & $99.86 \%$ & $-7.36 \%$ & $-1.51 \%$ & $12.63 \%$ \\
\hline DTLZ5(5) & $889.66 \%$ & $-49.83 \%$ & $17.88 \%$ & $679.59 \%$ & $-16.01 \%$ & $-42.34 \%$ \\
DTLZ5(10) & $36545.40 \%$ & $367006.47 \%$ & $781339.59 \%$ & $36207.13 \%$ & $111887.50 \%$ & $33493.00 \%$ \\
DTLZ5(15) & $78676.02 \%$ & $2590618.47 \%$ & $479086.99 \%$ & $142800.47 \%$ & $224323.88 \%$ & $39676.14 \%$ \\
\hline DTLZ6(5) & $1298.98 \%$ & $516.24 \%$ & $1380.03 \%$ & $388.15 \%$ & $339.36 \%$ & $284.90 \%$ \\
DTLZ6(10) & $4649.77 \%$ & $7734.03 \%$ & $1839.76 \%$ & $1104.26 \%$ & $2119.92 \%$ & $3342.06 \%$ \\
DTLZ6(15) & $5230.64 \%$ & $7423.63 \%$ & $1807.42 \%$ & $1830.18 \%$ & $1832.92 \%$ & $1441.22 \%$ \\
\hline DTLZ7(5) & $24.28 \%$ & $-8.00 \%$ & $17.98 \%$ & $-13.79 \%$ & $-9.39 \%$ & $7.00 \%$ \\
DTLZ7(10) & $73.94 \%$ & $1.38 \%$ & $538.91 \%$ & $31.77 \%$ & $-62.04 \%$ & $-32.85 \%$ \\
DTLZ7(15) & $172.12 \%$ & $89.95 \%$ & $1412.09 \%$ & $204.92 \%$ & $25.87 \%$ & $-8.31 \%$ \\
\hline Better/Worse & $21 / 0$ & $19 / 2$ & $20 / 1$ & $14 / 7$ & $12 / 9$ & $16 / 5$ \\
\hline
\end{tabular}

VaEA exists on $21,19,20,14,12$ and 16 test instances, respectively. In terms of NSGA-II, SPEA2, and PESA-II, SDR substantially improves their performance on almost all the MaOPs. Regarding GrEA, KnEA, and VaEA, SDR can also improve their performance especially on DTLZ1 and DTLZ3 with multi-modal landscapes and DTLZ5 and DTLZ6 with degenerate Pareto fronts, even if these three MOEAs were originally designed for manyobjective optimization.

Fig. 11 depicts the non-dominated set obtained by the six MOEAs based on Pareto dominance and SDR on 15-objective DTLZ3. It is clear that the MOEAs based on Pareto dominance cannot promote the population to converge to the global Pareto front of DTLZ3, even for the three MOEAs tailored for MaOPs. By contrast, the population obtained by all the SDR based MOEAs has significantly better convergence and diversity on DTLZ3. In conclusion, it is evidenced that SDR is a promising dominance relation for solving MaOPs.

\section{E. Effectiveness of the Adaptive Estimation of $\bar{\theta}$}

To verify the effectiveness of adaptive estimation of $\bar{\theta}$ in controlling the ratio of non-dominated solutions, we execute the NSGA-II/SDR on DTLZ1 and DTLZ5 with 3, 5 and 10 objectives. Here the population size is fixed to 100. Fig. 12 plots the ratios of non-dominated solutions identified by the proposed SDR during the optimization process, averaged over 30 runs. As shown in the figure, the ratio of non-dominated solutions is very close to 0.5 , which demonstrates the effectiveness of $\bar{\theta}$ in adaptively controlling the ratio of non-dominated solutions. Fig. 13 depicts the values of $\bar{\theta}$ over the optimization process, 

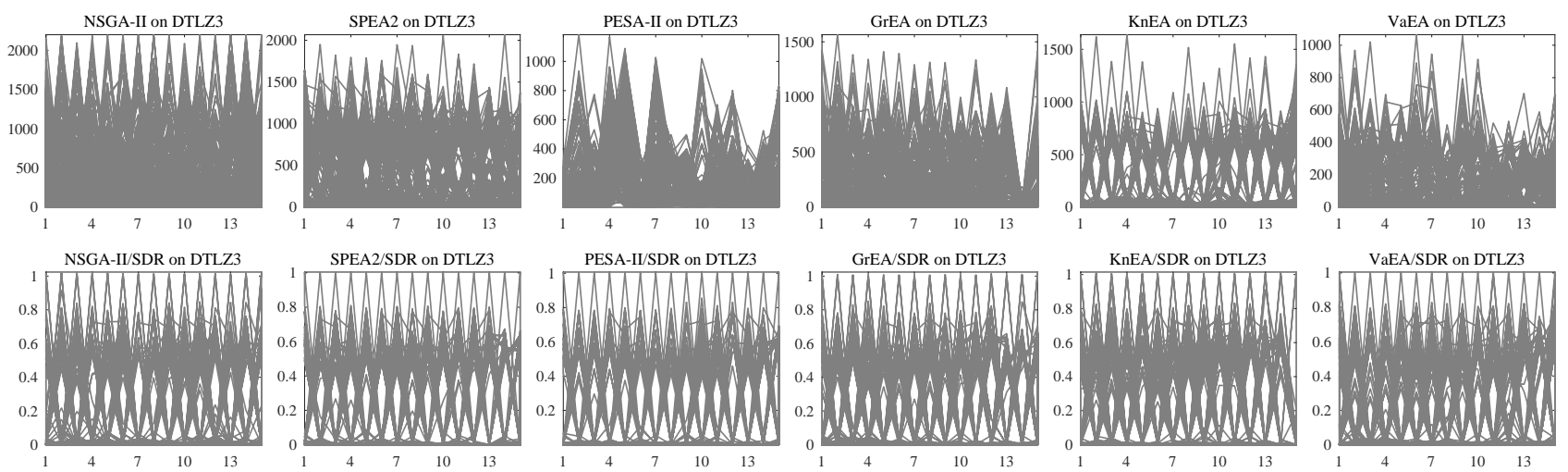

Fig. 11. Parallel coordinates of the non-dominated solution set with the median IGD among 30 runs obtained by NSGA-II, SPEA2, PESA-II, GrEA, KnEA, and VaEA based on Pareto dominance and SDR on 15-objective DTLZ3.
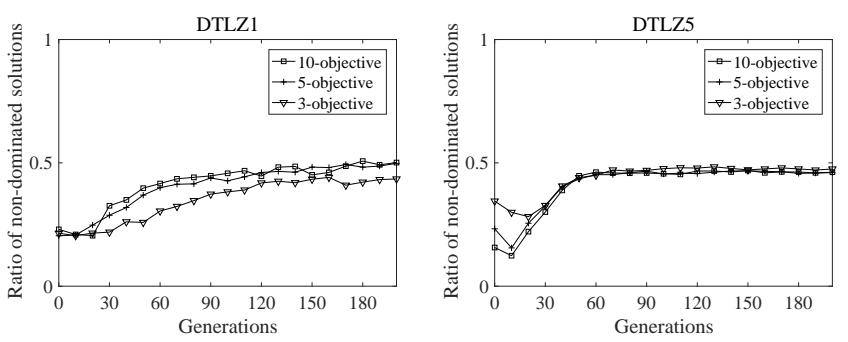

Fig. 12. The average ratios of non-dominated solutions during the optimization process of NSGA-II/SDR on DTLZ1 and DTLZ5 with 3, 5 and 10 objectives.

where it is clear that the values of $\bar{\theta}$ are distinct on different problems with different numbers of objectives. This is due to the fact that the distributions of the candidate solutions can be various when solving different problems. In particular, the Pareto front of $M$-objective DTLZ1 is an $(M-1)$-dimensional hyperplane, so the candidate solutions in high-objective space distribute much more sparsely than those in low-objective space. By contrast, since the Pareto front of DTLZ5 is always a degenerate one-dimensional curve, the candidate solutions show similar distributions regardless of the specific number of objectives. As a result, it is unreasonable to set $\bar{\theta}$ to a fixed value for solving different MaOPs; instead, the value of $\bar{\theta}$ should be adaptively adjusted during the optimization process, due to the various distributions of the candidate solutions.

\section{CONCLUSION AND Future WORK}

In this paper, a dominance relation has been proposed for many-objective optimization, termed strengthened dominance relation (SDR). The proposed SDR is effective in balancing the convergence and diversity of the non-dominated solution set, where it uses a niching technique based on the angles between the candidate solutions, and keeps only one candidate solution with the best convergence to be non-dominated in each niche. SDR is an adaptive method since the niche size is
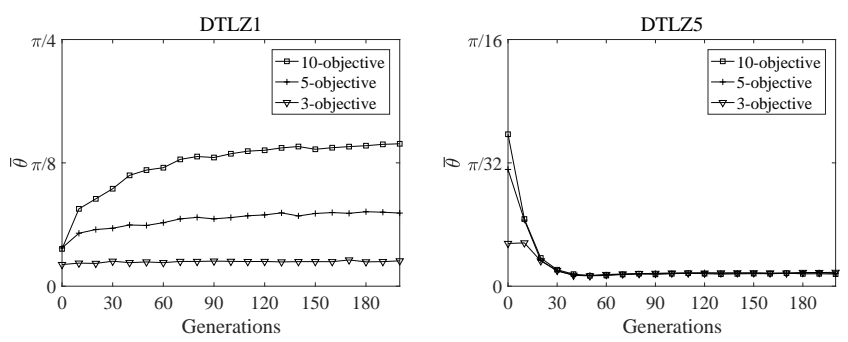

Fig. 13. The average values of $\bar{\theta}$ during the optimization process of NSGA-II/SDR on DTLZ1 and DTLZ5 with 3, 5 and 10 objectives.

automatically determined according to the distribution of the candidate solutions, which does not need to be predefined by users.

The comparison between SDR and eight existing dominance relations on several examples and benchmark problems has evidenced that SDR can better balance convergence and diversity in many-objective optimization. An MOEA, termed NSGA-II/SDR, is established based on SDR and NSGA-II, which has been empirically shown to be competitive to the state-of-the-art MOEAs for solving MaOPs. SDR has also been verified on six existing MOEAs, where the experimental results have evidenced that SDR can bring considerable improvement for these MOEAs in solving MaOPs.

Despite that NSGA-II/SDR performs fairly well on $\mathrm{MaOPs}$, it is known that the crowding distance is ineffective in solving MaOPs [55]. So it is desirable to further improve the performance of NSGA-II/SDR on MaOPs by developing a new MOEA which can effectively distinguish the non-dominated solutions identified by SDR. In addition, it is also interesting to assess the performance of SDR on real-world applications with many objectives [56], [57] in the future.

\section{REFERENCES}

[1] A. Zhou, B.-Y. Qu, H. Li, S.-Z. Zhao, P. N. Suganthan, and Q. Zhang, "Multiobjective evolutionary algorithms: A survey of the state of the art," Swarm and Evolutionary Computation, vol. 1, no. 1, pp. 32-49, 2011. 
[2] K. Deb, A. Pratap, S. Agarwal, and T. Meyarivan, "A fast and elitist multi-objective genetic algorithm: NSGA-II," IEEE Transactions on Evolutionary Computation, vol. 6, no. 2, pp. 182-197, 2002.

[3] D. E. Goldberg, Genetic algorithms in search, optimization, and machine learning. Boston, USA: Addison-Wesley, 1989.

[4] H. Ishibuchi, N. Tsukamoto, and Y. Nojima, “Evolutionary manyobjective optimization: A short review," in Proceedings of the 2008 IEEE Congress on Evolutionary Computation, 2008, pp. 2419-2426.

[5] Y. Tian, H. Wang, X. Zhang, and Y. Jin, "Effectiveness and efficiency of non-dominated sorting for evolutionary multiand many-objective optimization," Complex \& Intelligent Systems, vol. 3, no. 4, pp. 247-263, 2017.

[6] H. Sato, H. Aguirre, and K. Tanaka, "Controlling dominance area of solutions and its impact on the performance of MOEAs," in Proceedings of the 4th International Conference on Evolutionary MultiCriterion Optimization, 2007, pp. 5-20.

[7] C. Zhu, L. Xu, and E. D. Goodman, "Generalization of Paretooptimality for many-objective evolutionary optimization," IEEE Transactions on Evolutionary Computation, vol. 20, no. 2, pp. 299315, 2016.

[8] M. Laumanns, L. Thiele, K. Deb, and E. Zitzler, "Combining convergence and diversity in evolutionary multiobjective optimization," Evolutionary Computation, vol. 10, no. 3, pp. 263-282, 2002.

[9] S. Yang, M. Li, X. Liu, and J. Zheng, "A grid-based evolutionary algorithm for many-objective optimization," IEEE Transactions on Evolutionary Computation, vol. 17, no. 5, pp. 721-736, 2013.

[10] G. Wang and H. Jiang, "Fuzzy-dominance and its application in evolutionary many objective optimization," in Proceedings of the 2007 International Conference on Computational Intelligence and Security Workshops, 2007, pp. 195-198.

[11] Z. He, G. G. Yen, and J. Zhang, "Fuzzy-based Pareto optimality for many-objective evolutionary algorithms," IEEE Transactions on Evolutionary Computation, vol. 18, no. 2, pp. 269-285, 2014.

[12] Y. Yuan, H. Xu, B. Wang, and X. Yao, "A new dominance relationbased evolutionary algorithm for many-objective optimization," IEEE Transactions on Evolutionary Computation, vol. 20, no. 1, pp. 16-37, 2016.

[13] M. Elarbi, S. Bechikh, A. Gupta, L. B. Said, and Y. S. Ong, "A new decomposition-based NSGA-II for many-objective optimization," IEEE Transactions on Systems Man and Cybernetics: Systems, 2017, in press.

[14] X. Zhang, Y. Tian, and Y. Jin, "A knee point driven evolutionary algorithm for many-objective optimization," IEEE Transactions on Evolutionary Computation, vol. 19, no. 6, pp. 761-776, 2015.

[15] Y. Xiang, Y. Zhou, M. Li, and Z. Chen, "A vector angle based evolutionary algorithm for unconstrained many-objective optimization," IEEE Transactions on Evolutionary Computation, vol. 21, no. 1, pp. 131-152, 2017.

[16] J. Bader and E. Zitzler, "HypE: An algorithm for fast hypervolume-based many-objective optimization," Evolutionary Computation, vol. 19, no. 1, pp. 45-76, 2011.

[17] Y. Tian, R. Cheng, X. Zhang, F. Cheng, and Y. Jin, "An indicator based multi-objective evolutionary algorithm with reference point adaptation for better versatility," IEEE Transactions on Evolutionary Computation, 2017, in press.

[18] K. Li, K. Deb, Q. Zhang, and S. Kwong, "Combining dominance and decomposition in evolutionary many-objective optimization," IEEE Transactions on Evolutionary Computation, vol. 19, no. 5, pp. 694-716, 2015.

[19] M. Li, S. Yang, and X. Liu, "Pareto or non-Pareto: Bi-criterion evolution in multi-objective optimization," IEEE Transactions on Evolutionary Computation, vol. 20, no. 5, pp. 645-665, 2015.

[20] E. Zitzler, M. Laumanns, and L. Thiele, "SPEA2: Improving the strength Pareto evolutionary algorithm for multiobjective optimization," in Proceedings of the Fifth Conference on Evolutionary Methods for Design, Optimization and Control with Applications to Industrial Problems, 2001, pp. 95-100.

[21] D. W. Corne, N. R. Jerram, J. D. Knowles, and M. J. Oates, "PESA-II: Region-based selection in evolutionary multi-objective optimization," in Proceedings of the 2001 Conference on Genetic and Evolutionary Computation, 2001, pp. 283-290.

[22] X. Zhang, Y. Tian, R. Cheng, and Y. Jin, "An efficient approach to non-dominated sorting for evolutionary multi-objective optimization," IEEE Transactions on Evolutionary Computation, vol. 19, no. 2, pp. 201-213, 2015.
[23] _ - "A decision variable clustering-based evolutionary algorithm for large-scale many-objective optimization," IEEE Transactions on Evolutionary Computation, vol. 22, no. 1, pp. 97-112, 2018.

[24] H. Sato, H. Aguirre, and K. Tanaka, "Self-controlling dominance area of solutions in evolutionary many-objective optimization," Simulated Evolution and Learning, pp. 455-465, 2010.

[25] K. Ikeda, H. Kita, and S. Kobayashi, "Failure of Pareto-based MOEAs: Does non-dominated really mean near to optimal?" in Proceedings of the 2001 IEEE Congress on Evolutionary Computation, 2001, pp. 957-962.

[26] A. G. Hernández-Díaz, L. V. Santana-Quintero, C. A. C. Coello, and J. Molina, "Pareto-adaptive $\epsilon$-dominance," Evolutionary computation, vol. 15, no. 4, pp. 493-517, 2007.

[27] L. S. Batista and F. Campelo, "Pareto cone $\epsilon$-dominance: improving convergence and diversity in multiobjective evolutionary algorithms," in Proceedings of the 2011 International Conference on Evolutionary Multi-Criterion Optimization, 2011, pp. 76-90.

[28] M. Farina and P. Amato, "A fuzzy definition of "optimality" for many-criteria optimization problems," IEEE Transactions on Systems Man and Cybernetics - Part A Systems and Humans, vol. 34, no. 3, pp. 315-326, 2004.

[29] X. Zou, Y. Chen, M. Liu, and L. Kang, "A new evolutionary algorithm for solving many-objective optimization problems," IEEE Transactions on Systems, Man, and Cybernetics-Part B, vol. 38, no. 5, pp. 1402-1412, 2008.

[30] H. Ishibuchi, Y. Setoguchi, H. Masuda, and Y. Nojima, "Performance of decomposition-based many-objective algorithms strongly depends on Pareto front shapes," IEEE Transactions on Evolutionary Computation, vol. 21, no. 2, pp. 169-190, 2017.

[31] H. Wang, Y. Jin, and X. Yao, "Diversity assessment in manyobjective optimization," IEEE Transactions on Cybernetics, vol. 47, no. 6, pp. 1510-1522, 2017.

[32] M. Li, S. Yang, and X. Liu, "Diversity comparison of Pareto front approximations in many-objective optimization," IEEE Transactions on Cybernetics, vol. 44, no. 12, pp. 2568-2584, 2014.

[33] — " Bi-goal evolution for many-objective optimization problems," Artificial Intelligence, vol. 228, pp. 45-65, 2015.

[34] X. Ma, F. Liu, Y. Qi, X. Wang, L. Li, L. Jiao, M. Yin, and M. Gong, "A multiobjective evolutionary algorithm based on decision variable analyses for multiobjective optimization problems with largescale variables," IEEE Transactions on Evolutionary Computation, vol. 20, no. 2, pp. 275-298, 2016

[35] Y. Liu, D. Gong, J. Sun, and Y. Jin, "A many-objective evolutionary algorithm using a one-by-one selection strategy," IEEE Transactions on Cybernetics, vol. 47, no. 9, pp. 2689-2702, 2017.

[36] K. M. Clymont and E. Keedwell, "Deductive sort and climbing sort: New methods for non-dominated sorting," Evolutionary Computation, vol. 20, no. 1, pp. 1-26, 2012.

[37] R. Cheng, Y. Jin, K. Narukawa, and B. Sendhoff, "A multiobjective evolutionary algorithm using Gaussian process based inverse modeling," IEEE Transactions on Evolutionary Computation, vol. 19, no. 6, pp. 838-856, 2015.

[38] P. Weidner, "Advantages of hyperbola efficiency," in Multicriteria Decision, 1993, pp. 123-137.

[39] Q. Zhang and H. Li, "MOEA/D: A multi-objective evolutionary algorithm based on decomposition," IEEE Transactions on Evolutionary Computation, vol. 11, no. 6, pp. 712-731, 2007.

[40] Y. Tian, R. Cheng, X. Zhang, and Y. Jin, "PlatEMO: A MATLAB platform for evolutionary multi-objective optimization," IEEE Computational Intelligence Magazine, vol. 12, no. 4, pp. 73-87, 2017.

[41] V. Chankong and Y. Y. Haimes, Multiobjective Decision Making: Theory and Methodology. North-Holland, 1983.

[42] K. Deb and H. Jain, "An evolutionary many-objective optimization algorithm using reference-point based non-dominated sorting approach, part I: Solving problems with box constraints," IEEE Transactions on Evolutionary Computation, vol. 18, no. 4, pp. 577-601, 2014

[43] R. Cheng, Y. Jin, M. Olhofer, and B. Sendhoff, "A reference vector guided evolutionary algorithm for many-objective optimization," IEEE Transactions on Evolutionary Computation, vol. 20, no. 5, pp. 773-791, 2016.

[44] R. Hernández Gómez and C. A. Coello Coello, "Improved metaheuristic based on the R2 indicator for many-objective optimization," in Proceedings of the 2015 on Genetic and Evolutionary Computation Conference, 2015, pp. 679-686.

[45] K. Deb, Multi-Objective Optimization Using Evolutionary Algorithms. New York: Wiley, 2001 
[46] K. Deb and M. Goyal, "A combined genetic adaptive search (GeneAS) for engineering design," Computer Science and Informatics, vol. 26, no. 4, pp. 30-45, 1996.

[47] K. Deb, L. Thiele, M. Laumanns, and E. Zitzler, "Scalable test problems for evolutionary multiobjective optimization," in Evolutionary Multiobjective Optimization. Theoretical Advances and Applications, 2005, pp. 105-145.

[48] L. B. S. Huband, P. Hingston and L. While, "A review of multiobjective test problems and a scalable test problem toolkit," IEEE Transactions on Evolutionary Computation, vol. 10, no. 5, pp. 477506, 2006.

[49] H. Jain and K. Deb, "An evolutionary many-objective optimization algorithm using reference-point based nondominated sorting approach, part II: Handling constraints and extending to an adaptive approach," IEEE Transactions on Evolutionary Computation, vol. 18, no. 4, pp. 602-622, 2014.

[50] M. Li, C. Grosan, S. Yang, X. Liu, and X. Yao, "Multi-line distance minimization: A visualized many-objective test problem suite," IEEE Transactions on Evolutionary Computation, 2017, in press.

[51] K. Deb and D. K. Saxena, "On finding Pareto-optimal solutions through dimensionality reduction for certain largedimensional multi-objective optimization problems," Kangal report, vol. 2005011, 2005.

[52] A. Zhou, Y. Jin, Q. Zhang, B. Sendhoff, and E. Tsang, "Combining model-based and genetics-based offspring generation for multiobjective optimization using a convergence criterion," in Proceedings of the 2006 IEEE Congress on Evolutionary Computation, 2006, pp. 892-899.

[53] L. While, P. Hingston, L. Barone, and S. Huband, "A faster algorithm for calculating hypervolume," IEEE Transactions on Evolutionary Computation, vol. 10, no. 1, pp. 29-38, 2006.

[54] M. Li, L. Zhen, and X. Yao, "How to read many-objective solution sets in parallel coordinates [educational forum]," IEEE Computational Intelligence Magazine, vol. 12, no. 4, pp. 88-100, 2017.

[55] M. Li, S. Yang, and X. Liu, "Shift-based density estimation for Pareto-based algorithms in many-objective optimization," IEEE Transactions on Evolutionary Computation, vol. 18, no. 3, pp. 348$365,2014$.

[56] R. Cheng, T. Rodemann, M. Fischer, M. Olhofer, and Y. Jin, "Evolutionary many-objective optimization of hybrid electric vehicle control: From general optimization to preference articulation," IEEE Transactions on Emerging Topics in Computational Intelligence, vol. 1, no. 2, pp. 97-111, 2017.

[57] S. Nguyen, Y. Mei, and M. Zhang, "Genetic programming for production scheduling: a survey with a unified framework," Complex E Intelligent Systems, vol. 3, pp. 41-66, 2017.

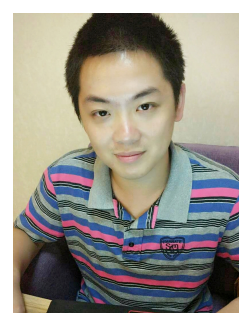

Ye Tian received the B.Sc., M.Sc., and Ph.D. degrees from Anhui University, Hefei, China, in 2012, 2015, and 2018, respectively.

He is currently a Lecture with the Institute of Physical Science and Information Technology, Anhui University, Hefei, China. His current research interests include multi-objective optimization methods and their application. He is the recipient of the 2017 IEEE Transactions on Evolutionary Computation Outstanding Paper Award.

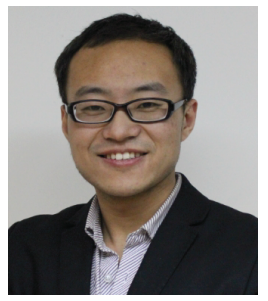

Ran Cheng (M'16) received the B.Sc. degree from the Northeastern University, Shenyang, China, in 2010, and the Ph.D. degree from the University of Surrey, Guildford, U.K., in 2016.

He is currently an Assistant Professor with the Department of Computer Science and Engineering, Southern University of Science and Technology, Shenzhen, China. His current research interests include evolutionary multiobjective optimization, model-based evolutionary algorithms, large-scale optimization, swarm intelligence, and deep learning. He is the founding chair of IEEE Symposium on Model Based Evolutionary Algorithms (IEEE MBEA). He is the recipient of the 2017 IEEE Transactions on Evolutionary Computation Outstanding Paper Award, and the 2019 IEEE Computational Intelligence Society (CIS) Outstanding Ph.D. Dissertation Award.

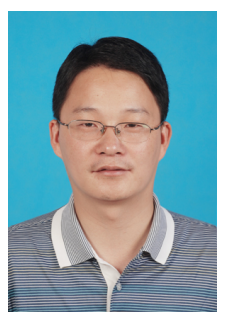

Xingyi Zhang received the B.Sc. degree from Fuyang Normal College, Fuyang, China, in 2003, and the M.Sc. and Ph.D. degrees from Huazhong University of Science and Technology, Wuhan, China, in 2006 and 2009, respectively.

He is currently a Professor with the School of Computer Science and Technology, Anhui University, Hefei, China. His current research interests include unconventional models and algorithms of computation, multi-objective optimization, and membrane computing. He is the recipient of the 2017 IEEE Transactions on Evolutionary Computation Outstanding Paper Award.

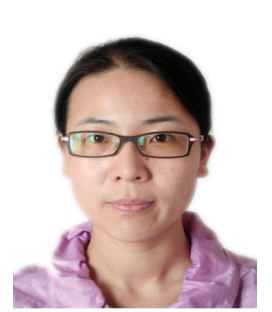

Yansen Su received the B.Sc. degree from Tangshan Normal University, Tangshan, China, in 2007, the M.Sc. degree from Shandong University of Science and Technology, Qingdao, China, in 2010, and the Ph.D. degree from Huazhong University of Science and Technology, Wuhan, China, in 2014.

She is currently an Associate Professor in the School of Computer Science and Technology, Anhui University, Hefei, China. Her main research interests include complexes networks, computational biology, and multi-objective optimization.

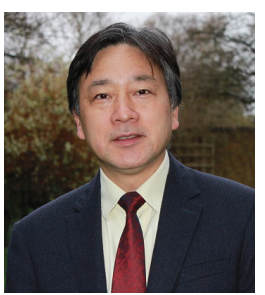

Yaochu Jin (M'98-SM'02-F'16) received the B.Sc., M.Sc., and Ph.D. degrees from Zhejiang University, Hangzhou, China, in 1988, 1991, and 1996, respectively, and the Dr.-Ing. degree from Ruhr University Bochum, Bochum, Germany, in 2001.

He is currently a Professor in Computational Intelligence, Department of Computer Science, University of Surrey, Guildford, U.K., where he heads the Nature Inspired Computing and Engineering Group. He is also a Finland Distinguished Professor and a Changiiang Distinguished Visiting Professor appointed. He has (co)authored over 200 peer-reviewed journal and conference papers and been granted eight patents on evolutionary optimization.

He is the Editor-in-Chief of the IEEE Transactions on Cognitive and Developmental Systems and Complex \& Intelligent Systems. He is also an Associate Editor or Editorial Board Member of the IEEE Transactions on Evolutionary Computation, IEEE Transactions on Cybernetics, IEEE Transactions on Nanobioscience, Evolutionary Computation, BioSystems, Soft Computing, and Natural Computing. He is an IEEE Distinguished Lecturer (2017-2019). He is the recipient of the 2014 and 2016 IEEE Computational Intelligence Magazine Outstanding Paper Award, the 2017 IEEE Transactions on Evolutionary Computation Outstanding Paper Award, and the Best Paper Award of the 2010 IEEE Symposium on Computational Intelligence in Bioinformatics and Computational Biology. He is a Fellow of IEEE. 